\title{
A robust beamformer design for underlay cognitive radio networks using worst case optimization
}

\author{
Uditha Lakmal Wijewardhana ${ }^{1 *}$, Marian Codreanu ${ }^{1}$, Matti Latva-aho ${ }^{1}$ and Anthony Ephremides ${ }^{2}$
}

\begin{abstract}
We propose a robust beamforming design for underlay cognitive radio networks where multiple secondary transmitters communicate with corresponding secondary receivers and coexist with a primary network. We consider a scenario where all transmitters have multiple antennas and all primary and secondary receivers are equipped with a single antenna. The main focus is to design the optimal transmit beamforming vectors for secondary transmitters that maximize the minimum of the received signal-to-interference-plus-noise ratios of the cognitive users. The interference powers to the primary receivers are kept below a threshold to guarantee that the performance of the primary network does not degrade due to the secondary network. Imperfect channel state information (CSI) in all relevant channels are considered, and a bounded ellipsoidal uncertainty model is used to model the CSI errors. We recast the problem in the form of semidefinite program and an iterative algorithm based on the bisection method is proposed to achieve the optimal solution. Further, we propose upper and lower bounds for the optimal value of the considered problem, which provides better initialization for the algorithm. Numerical simulations are conducted to show the effectiveness of the proposed method against the non-robust design.
\end{abstract}

Keywords: Cognitive radio networks; Optimization; Robust beamforming design; Semidefinite programming (SDP); Semidefinite relaxation

\section{Introduction}

Scarcity of wireless spectrum is one of the major challenges faced by the modern wireless communication industry. Due to rapid deployment of wireless services in the recent past and the fixed spectrum allocation policy, the wireless spectrum has been increasingly crowded. On the other hand, according to the Federal Communication Commissions and other regulatory bodies, most of the allocated spectrum is under utilized [1]. Therefore, the secondary usage of wireless spectrum has been proposed as a method to utilize more efficiently the wireless spectrum [1-4].

Cognitive radio networks (CRNs) [2-4] operate on this idea of the secondary usage of spectrum. Here, the secondary network is allowed to opportunistically access the spectrum owned by the primary network provided that

*Correspondence: uditha@ee.oulu.fi

${ }^{1}$ Centre for Wireless Communications, University of Oulu, P.O. Box 4500, Oulu 90014, Finland

Full list of author information is available at the end of the article it does not degrade the performance of the primary network. Hence, there are two major challenges that should be addressed by a CRN. The first challenge is to maximize the performance of the users in the CRN as much as possible. The second challenge is to guarantee the performance or the quality of service (QoS) of the primary network, which is the main constraint in a CRN. Specifically, in underlay CRNs, the performance of the secondary (cognitive) users should be maximized while the maximum interference power to the primary users (PUs) should remain below a pre-specified threshold [4].

Resource allocation problem for underlay CRNs have been studied recently in [5-9], assuming that perfect channel state information (CSI) knowledge for all relevant links is available for the design. Beamformer design for multiple-input-multiple-output (MIMO) ad hoc CRN is presented in [5]. The weighted sum-rate of the CRN is maximized in [5] subject to the individual power constraints and interference constraint to the coexisting PU. A semi-distributed algorithm is proposed to

\section{贷 Springer}

(c) 2014 Wijewardhana et al: licensee Springer. This is an Open Access article distributed under the terms of the Creative

Commons Attribution License (http://creativecommons.org/licenses/by/2.0), which permits unrestricted use, distribution, and reproduction in any medium, provided the original work is properly cited. 
achieve a locally optimal solution and an alternative centralized algorithm has been proposed based on geometric programming and network duality. A game theoretic approach for the same problem with a CRN that coexists with multiple PUs is presented in [6].

However, in practice, the channel vectors are estimated from training sequences and, inherently, this leads to imperfect estimation. These CSI estimation errors can greatly affect the performance of the network, resulting in degradation in both primary and secondary users' QoS. Such channel errors are usually modeled either by a bounded uncertainty model such as D-norm, polyhedron, ellipsoidal [10] or a stochastic error model [11].

Robust beamformer design with imperfect CSI has received a considerable attention recently. Usually, this problem is tackled by either worst-case optimization [12-27] or stochastic optimization [24,28]. In worst-case optimization (or maximin optimization), the uncertain parameters can take some given set of possible values, but without any known distribution. Then the optimization variables are designed in such a way that an objective value is maximized while guaranteeing the feasibility of the constraints over the given set of possible values of the parameters. This method has been applied to design the robust beamforming vectors for underlay CRNs in [2026], where the channel errors are either norm bounded or bounded by ellipsoids. With the exception of [24] and [26], most of the abovementioned work consider a CRN where a single secondary transmitter (TX) co-exists with a primary network. The problem of maximizing the minimum signal-to-interference-plus-noise ratio (SINR) in an underlay CRN, where the transmitter communicates with multiple secondary receivers (RXs) is studied in [21]. An iterative solution has been proposed based on semidefinite relaxation [29], and if the solution is not rank-one, rank-one approximations [29] have to be used to achieve the beamforming vectors. For the same problem, a method to achieve a rank-one solution with some tolerance is presented in [22]. Therefore, none of the above work guarantee the optimal solution of the problem of maximizing the minimum SINR in an underlay CRN.

The sum mean square error is minimized in [24] for an underlay MIMO ad hoc CRN constrained to individual power budgets of secondary TXs, where the channel errors are bounded by Euclidean balls. There the authors have cast the problem as a semidefinite program (SDP) and solved iteratively via standard interior point methods to achieve a suboptimal solution for the problem. In [26], the same problem of minimizing the sum mean square error for an underlay MIMO ad hoc CRN subject to individual power budgets for the secondary TXs was considered. A distributed solution was proposed under the assumption that secondary TXs have perfect CSI knowledge of the channels to the secondary RXs. Furthermore, the SINR of an underlay MIMO secondary link is maximized in [30] constrained to the power budget of the secondary TX under the same assumption that secondary TX has perfect CSI knowledge of the channel to the secondary RX.

The focus of this paper is to design the optimal robust beamforming vectors for the secondary TXs in a CRN that coexists with a primary network. We address the problem of maximizing the worst SINR of any secondary user subject to interference constraints to primary network and individual power constraints. Further, we assume that the network controller has imperfect CSI knowledge and all relevant channel vectors may take any value within some pre-specified bounded uncertainty ellipsoids $[10,14,17]$. An equivalent reformulation of the problem is obtained, and then the S-procedure $[31,32]$ is used to handle the non-convex quadratic constraints due to channel uncertainties. In particular, we replace each quadratic constraint pair by a linear matrix inequality (LMI) for a fixed objective value by means of the S-lemma $[18,21]$, which leads to a SDP. An iterative algorithm based on the bisection method is proposed to solve the relaxed version (relaxed the non-convex rank constraints in the problem) of the reformulated problem. Finally, we show that the optimal solution for the original problem can be achieved by introducing a properly chosen objective function to the feasibility check step of the iterative algorithm.

As the main contribution, in this work, we show the ability to handle CSI uncertainty in a multiple-input-singleoutput (MISO) communication network with interference temperature constraints. Further, we provide a rigorous proof for the tightness of SDP relaxation in worst SINR maximization problem in multi-cell downlink scenario with a single user per cell and subject to interference temperature constraints. Additionally, we show that the proposed solution approach can be extended to maximize the worst weighted SINR or, more generally, the worst among a set of increasing functions of each SINR.

Organization: Section 2 describes the network and the channel uncertainty models used in this paper. The problem formulation for the worst-case scenario and a suitable equivalent reformulation is presented in Section 3. In Section 4, the SDP-based solution is presented and an iterative algorithm is proposed to obtain the optimal beamforming vectors. Simulation results are presented in Section 5. Finally, we conclude the paper with Section 6.

Notations: Throughout this paper, $\mathbb{C}^{l}, \mathbb{H}^{l}$ and $\mathbb{R}$ denotes the set of $l$-dimensional complex vectors, the set of $l$ dimensional complex Hermitian matrices and the set of real values, respectively. Further, the complex column vectors and matrices are represented by the boldfaced lowercase and uppercase letters, respectively, e.g. $\mathbf{w}$ and $\mathbf{W}$. We denote a real scalar by a lower case letter and $\operatorname{Re}(\cdot)$ denotes the real part of a complex number. The superscript $(\cdot)^{\mathrm{T}}$ 
denotes the transpose and $(\cdot)^{\mathrm{H}}$ denotes the Hermitian (conjugate transpose) operation for a vector or a matrix. $\mathbf{W} \succeq 0$ and $\mathbf{W} \succ 0$ means that $\mathbf{W}$ is positive semidefinite and positive definite, respectively. Rank and the trace of a matrix are represented by $\operatorname{Rank}(\mathbf{W})$ and $\operatorname{Trace}(\mathbf{W})$, respectively. Modulus of a scalar is denoted by $|\cdot|$ and $\|\cdot\|$ denotes the Euclidean norm of a vector. The expectation of a random variable is denoted by $E\{\cdot\}$. In addition, $\mathbf{I}_{n}$ denotes the $n$-dimensional identity matrix while $\mathbf{0}$ denotes an all-zero vector or matrix with appropriate dimensions. We denote the complex normal distribution with mean $\mu$ and covariance $\sigma^{2}$ by $\mathcal{C N}\left(\mu, \sigma^{2}\right)$. The distribution $\mathcal{T C N}\left(\mu, \sigma^{2}, a, b\right)$ represents the truncated complex normal distribution with parameters $\mu, \sigma^{2}, a$ and $b$ (see [33], Chap. 4 in [34]). Finally, in an optimization problem, if $w$ is a variable, then $w^{\star}$ denotes the optimal value or the optimal solution.

\section{System model}

We provide the detailed description of the considered system model in this section. Specifically, we describe the network model and the channel uncertainty model used throughout the paper.

\subsection{Network model}

We consider an underlay CRN consisting of multiple transmitter-receiver pairs which coexists with a primary network. The network model is shown in Figure 1. The set of secondary links is denoted by $\mathcal{N}$ and we label them as $n=1, \ldots, N$. We use the same indexing for the secondary transmitters and receivers, i.e., we refer to the transmitter (receiver) of the $n$th secondary link as the $n$th secondary TX (RX). We consider a MISO downlink scenario and assume that each secondary TX (a base station) is equipped with $n_{t}$ antennas to communicate with its corresponding secondary RX (a mobile station). We

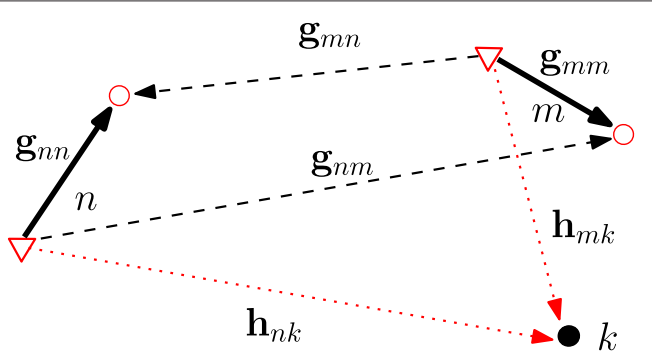

\section{Secondary TX O Secondary RX • Primary RX}

Figure 1 System model. Underlay cognitive radio network with two secondary TX-RX pairs $(N=2)$ coexists with a primary network consisting a single primary $\mathrm{RX}(K=1)$. Straight arrows show the desired channels and the dashed arrows show the interfering channels. represent the set of all primary RXs by $\mathcal{K}$ and label them as $k=1, \ldots, K$. Further, we assume that all the primary and secondary RXs are equipped with a single antenna ${ }^{\mathrm{a}}$.

We assume that all secondary TXs operate in the same frequency band as the primary network and use transmit beamforming to communicate with their corresponding secondary RXs. We further assume that a network controller decides the resource allocation and beamforming vectors for each secondary TX.

Single-stream beamforming transmission strategy is assumed and hence the signal transmitted by the $n$th secondary TX is given by

$$
\mathbf{x}_{n}=\mathbf{w}_{n} d_{n},
$$

where $d_{n}$ denotes the (complex) information symbol and $\mathbf{w}_{n}$ is its associated beamforming vector. We assume that the information symbols are independent, i.e., $E\left\{d_{n} d_{m}^{H}\right\}=$ 0 for all $m \neq n, n, m \in \mathcal{N}$, and normalized such that $E\left\{\left|d_{n}\right|^{2}\right\}=1$ for all $n \in \mathcal{N}$. Therefore, the transmit power of the $n$th secondary TX is given by $\left\|\mathbf{w}_{n}\right\|^{2}$ and it is limited by $P_{\max }$, the maximum available transmit power, for all $n \in \mathcal{N}$.

We denote the channel vector from the $m$ th secondary TX to the $n$th secondary RX by $\mathbf{g}_{m n} \in \mathbb{C}^{n_{t}}$. The signal received at the $n$th secondary RX can be written as

$$
y_{n}=\mathbf{g}_{n n}^{H} \mathbf{w}_{n} d_{n}+\sum_{\substack{m=1 \\ m \neq n}}^{N} \mathbf{g}_{m n}^{H} \mathbf{w}_{m} d_{m}+z_{n},
$$

where the first term is the signal of interest, the second term represents the interference from the secondary network and $z_{n} \in \mathbb{C}$ is the additive noise at the $n$th secondary RX. We assume that the noise term $z_{n}$ has power $\sigma_{n}^{2}$ and it includes the receiver's thermal noise and the interference from the primary network. Therefore, the instantaneous SINR at the $n$th secondary RX can be expressed as

$$
\operatorname{SINR}_{n}=\frac{\left|\mathbf{g}_{n n}^{H} \mathbf{w}_{n}\right|^{2}}{\sum_{\substack{m=1 \\ m \neq n}}^{N}\left|\mathbf{g}_{m n}^{H} \mathbf{w}_{m}\right|^{2}+\sigma_{n}^{2}} .
$$

The interfering channel from the $n$th secondary TX to the $k$ th primary RX is denoted by $\mathbf{h}_{n k} \in \mathbb{C}^{n_{t}}$. Now, the total interference power caused by the secondary network on the $k$ th primary $\mathrm{RX}$ can be written as

$$
I_{k}=\sum_{n=1}^{N}\left|\mathbf{h}_{n k}^{H} \mathbf{w}_{n}\right|^{2},
$$

which should be limited by the interference threshold $I_{\text {th }}$ in order to guarantee the QoS of the primary network.

\subsection{Channel uncertainty model}

We assume that the channels are uncertain at the network controller $^{\mathrm{b}}$, but they belong to a known compact sets of 
possible values. Specifically, we assume that the channel vectors, $\mathbf{g}_{m n}$ and $\mathbf{h}_{n k}$ for all $n, m \in \mathcal{N}$ and $k \in \mathcal{K}$, belong to known ellipsoidal uncertainty sets.

We model the channel vector, $\mathbf{g}_{m n}$, from the $m$ th secondary TX to the $n$th secondary RX as the sum of two components, i.e.,

$$
\mathbf{g}_{m n}=\hat{\mathbf{g}}_{m n}+\mathbf{e}_{m n},
$$

where $\hat{\mathbf{g}}_{m n} \in \mathbb{C}^{n_{t}}$ denotes the estimated value at the network controller and $\mathbf{e}_{m n}$ represents the corresponding channel estimation error. It is assumed that $\mathbf{e}_{m n}$ can take any value inside a $n_{t}$-dimensional complex ellipsoid. Hence, the channel uncertainty set can be defined as

$$
\mathcal{E}_{m n}\left(\mathbf{Q}_{m n}\right)=\left\{\mathbf{e}_{m n}: \mathbf{e}_{m n}^{H} \mathbf{Q}_{m n} \mathbf{e}_{m n} \leq 1\right\},
$$

where $\mathbf{Q}_{m n}$ is a complex Hermitian positive definite matrix $\left(\mathbf{Q}_{m n} \succ 0\right)$, assumed to be known, which specifies the size and shape of the ellipsoid. For example, when $\mathbf{Q}_{m n}=\left(1 / \xi_{m n}^{2}\right) \mathbf{I}$, the ellipsoidal channel error model (6) reduces to $\left\|\mathbf{e}_{m n}\right\|^{2} \leq \xi_{m n}^{2}$. This represents a ball uncertainty region [35] with uncertainty radius $\xi_{m n}$. In this model, $\xi_{m n}=0$ implies that perfect CSI knowledge is considered and with $\xi_{m n}$ the channel uncertainty becomes larger causing CSI knowledge to become imperfect [21].

We use the same uncertainty model for the channel vector, $\mathbf{h}_{n k}$, from the $n$th secondary TX to the $k$ th primary RX, i.e.,

$$
\mathbf{h}_{n k}=\hat{\mathbf{h}}_{n k}+\tilde{\mathbf{e}}_{n k}
$$

where $\hat{\mathbf{h}}_{n k} \in \mathbb{C}^{n_{t}}$ is the estimated value at the network controller and $\tilde{\mathbf{e}}_{n k}$ denotes the corresponding channel estimation error. The ellipsoidal channel uncertainty set can be defined as

$$
\tilde{\mathcal{E}}_{n k}\left(\tilde{\mathbf{Q}}_{n k}\right)=\left\{\tilde{\mathbf{e}}_{n k}: \tilde{\mathbf{e}}_{n k}^{H} \tilde{\mathbf{Q}}_{n k} \tilde{\mathbf{e}}_{n k} \leq 1\right\},
$$

where $\tilde{\mathbf{Q}}_{n k} \succ 0$ specifies the size and shape of the uncertainty ellipsoid.

\section{Problem formulation}

Our objective is to maximize the performance of the CRN while satisfying the QoS requirements of the primary network. We consider the minimum SINR among all secondary receivers as the performance indicator of the CRN and the interference received from the CRN as the QoS measurement for the primary network. Then the solution of the problem will guarantee a certain SINR for all secondary RXs while the interference to all the RXs in the primary network will be lower than a predefined threshold $I_{\text {th }}$. The mathematical formulation of the mentioned prob- lem and a suitable equivalent reformulation is presented in this section.

\subsection{Problem formulation}

Now, with the channel uncertainty model above, we can re-write the instantaneous SINR at the $n$th secondary RX as

$$
\operatorname{SINR}_{n}=\frac{\left|\left(\hat{\mathbf{g}}_{n n}+\mathbf{e}_{n n}\right)^{H} \mathbf{w}_{n}\right|^{2}}{\sum_{\substack{m=1 \\ m \neq n}}^{N}\left|\left(\hat{\mathbf{g}}_{m n}+\mathbf{e}_{m n}\right)^{H} \mathbf{w}_{m}\right|^{2}+\sigma_{n}^{2}},
$$

and the interference power, caused by the secondary network on the $k$ th primary $\mathrm{RX}$ as

$$
I_{k}=\sum_{n=1}^{N}\left|\left(\hat{\mathbf{h}}_{n k}+\tilde{\mathbf{e}}_{n k}\right)^{H} \mathbf{w}_{n}\right|^{2} .
$$

The resource allocation problem we address in this work is to optimize the transmit beamforming vectors in CRN, $\left\{\mathbf{w}_{n}\right\}_{n=1}^{N}$, to maximize the minimum SINR of the secondary RXs for given parameters $P_{\max }$ and $I_{\text {th }}$. Due to the CSI uncertainty, the beamfomer design should guarantee a certain SINR for any channel error value inside the uncertainty region. Further, the design should keep the interference to the primary RXs below the threshold for all channel errors inside the uncertainty region to guarantee the performance of the primary network. This problem can be mathematically expressed as

$$
\begin{array}{ll}
\operatorname{maximize} & \min _{n=1, \ldots, N} \inf _{\mathbf{e}_{m n} \in \mathcal{E}_{m n}, m \in \mathcal{N}} \operatorname{SINR}_{n} \\
\text { subject to } & \sup _{\tilde{e}_{n k} \in \tilde{\mathcal{E}}_{n k}, n \in \mathcal{N}} I_{k} \leq I_{\text {th }}, \quad k \in \mathcal{K} \\
& \left\|\mathbf{w}_{n}\right\|_{2}^{2} \leq P_{\max } \quad n \in \mathcal{N},
\end{array}
$$

where the optimization variables are $\mathbf{w}_{n}, \mathbf{e}_{m n}, \tilde{\mathbf{e}}_{n k}$ for $n, m \in \mathcal{N}, k \in \mathcal{K}$. Note that $\operatorname{SINR}_{n}$ depends on $\mathbf{e}_{m n}$ for all $m \in \mathcal{N}$ (see (9)) and $I_{k}$ depends on $\tilde{\mathbf{e}}_{n k}$ for all $n \in \mathcal{N}$ (see (10)). In Problem (11), the infimum in the objective function and supremum in the first constraint are taken over all possible channel errors contained in the given uncertainty region.

\subsection{An equivalent reformulation}

Since the minimization in the objective function of Problem (11) is over all secondary RXs, the optimal value should be less than or equal to any SINR value that can be achieved by a secondary RX for the optimal beamformer design. Therefore, the optimization Problem (11) can be equivalently written in the epigraph form (see 
p. 134 in [31]) (strictly speaking, this is a hypograph form, as Problem (11) is a maximization) as ${ }^{\mathrm{c}}$

maximize $\gamma$

$$
\begin{gathered}
\text { subject to } \gamma \leq \frac{\left|\left(\hat{\mathbf{g}}_{n n}+\mathbf{e}_{n n}\right)^{H} \mathbf{w}_{n}\right|^{2}}{\sum_{\substack{m=1 \\
m \neq n}}^{N}\left|\left(\hat{\mathbf{g}}_{m n}+\mathbf{e}_{m n}\right)^{H} \mathbf{w}_{m}\right|^{2}+\sigma_{n}^{2}}, \\
\forall \mathbf{e}_{m n} \in \mathcal{E}_{m n}, \quad n \in \mathcal{N} \\
\sum_{n=1}^{N}\left|\left(\hat{\mathbf{h}}_{n k}+\tilde{\mathbf{e}}_{n k}\right)^{H} \mathbf{w}_{n}\right|^{2} \leq I_{\mathrm{th}}, \\
\forall \tilde{\mathbf{e}}_{n k} \in \tilde{\mathcal{E}}_{n k}, k \in \mathcal{K} \\
\left\|\mathbf{w}_{n}\right\|_{2}^{2} \leq P_{\max }, n \in \mathcal{N},
\end{gathered}
$$

where the optimization variables are $\gamma$ and $\mathbf{w}_{n}, \mathbf{e}_{m n}, \tilde{\mathbf{e}}_{n k}$ for $n, m \in \mathcal{N}, k \in \mathcal{K}$.

By introducing the new variables,

$$
\begin{aligned}
& s_{m n}=\left|\left(\hat{\mathbf{g}}_{m n}+\mathbf{e}_{m n}\right)^{H} \mathbf{w}_{m}\right|^{2}, \quad m \in \mathcal{N} \backslash\{n\}, n \in \mathcal{N} \\
& I_{n k}=\left|\left(\hat{\mathbf{h}}_{n k}+\tilde{\mathbf{e}}_{n k}\right)^{H} \mathbf{w}_{n}\right|^{2}, \quad n \in \mathcal{N}, k \in \mathcal{K}
\end{aligned}
$$

Problem (12) can be recast equivalently as

$$
\begin{aligned}
\text { maximize } & \gamma \\
\text { subject to } & \left(\hat{\mathbf{g}}_{n n}+\mathbf{e}_{n n}\right)^{H} \mathbf{w}_{n} \mathbf{w}_{n}^{H}\left(\hat{\mathbf{g}}_{n n}+\mathbf{e}_{n n}\right) \\
& \geq \gamma\left(\sum_{\substack{m=1 \\
m \neq n}}^{N} s_{m n}+\sigma_{n}^{2}\right), \forall \mathbf{e}_{n n} \in \mathcal{E}_{n n}, n \in \mathcal{N} \\
& \left(\hat{\mathbf{g}}_{m n}+\mathbf{e}_{m n}\right)^{H} \mathbf{w}_{m} \mathbf{w}_{m}^{H}\left(\hat{\mathbf{g}}_{m n}+\mathbf{e}_{m n}\right) \leq s_{m n}, \\
& \forall \mathbf{e}_{m n} \in \mathcal{E}_{m n}, m \in \mathcal{N} \backslash\{n\}, n \in \mathcal{N} \\
& \sum_{n=1}^{N} I_{n k} \leq I_{\mathrm{th}}, \quad k \in \mathcal{K} \\
& (15 \mathrm{c}) \\
& \left(\hat{\mathbf{h}}_{n k}+\tilde{\mathbf{e}}_{n k}\right)^{H} \mathbf{w}_{n} \mathbf{w}_{n}^{H}\left(\hat{\mathbf{h}}_{n k}+\tilde{\mathbf{e}}_{n k}\right) \leq I_{n k}, \\
& \forall \tilde{\mathbf{e}}_{n k} \in \tilde{\mathcal{E}}_{n k}, n \in \mathcal{N}, k \in \mathcal{K} \quad(15 \mathrm{~d}) \\
& \mathbf{w}_{n}^{H} \mathbf{w}_{n} \leq P_{\max }, \quad n \in \mathcal{N}, \quad(15 \mathrm{f})
\end{aligned}
$$

where the optimization variables are $\gamma$ and $\mathbf{w}_{n}, \mathbf{e}_{m n}, \tilde{\mathbf{e}}_{n k}, s_{m n}, I_{n k}$ for all $n, m \in \mathcal{N}, k \in \mathcal{K}$. Note that we have re-written 1st constraint in Problem (12) as two separate ones, i.e., (15b) and (15c). Furthermore, it is easy to show (e.g., by contradiction) that constraints (15c) and (15e) are tight (i.e., they hold with equality at optimality). Hence, Problem (15) is an equivalent reformulation of Problem (12).

\section{Optimal beamformer design}

In this section, we propose an iterative algorithm to solve the equivalent Problem (15) and compute the optimal beamforming vectors for the underlay CRN.

\subsection{Optimal solution and iterative algorithm}

The outer product $\mathbf{w}_{n} \mathbf{w}_{n}^{H}$ in Problem (15) is a rank-one positive semidefinite matrix. We introduce a new set of variables $\mathbf{W}_{n}=\mathbf{w}_{n} \mathbf{w}_{n}^{H}$ for all $n \in \mathcal{N}$. Then the constraints (15b), (15c), (15d) and (15f) become linear in $\mathbf{W}_{n}$, and $\mathbf{W}_{n}$ should be rank one. Furthermore, we can see that the constraint (15b) is quadratic in $\mathbf{e}_{n n}$, constraint (15c) is quadratic in $\mathbf{e}_{m n}$ and (15e) is quadratic in $\tilde{\mathbf{e}}_{n k}$. This suggests that we can use the following lemma to recast these constraints in such a way that they become linear matrix inequalities (LMIs) for fixed value of $\gamma$ in Problem (15).

$S$-lemma[18,31,32]: Let $\Phi_{i}$ be a real valued function of an $l$-dimensional complex vector $\mathbf{y}$, defined as

$$
\Phi_{i}(\mathbf{y})=\mathbf{y}^{H} \mathbf{A}_{i} \mathbf{y}+2 \operatorname{Re}\left(\mathbf{b}_{i}^{H} \mathbf{y}\right)+c_{i}
$$

where $\mathbf{A}_{i} \in \mathbb{H}^{l}, \mathbf{b}_{i} \in \mathbb{C}^{l}, c_{i} \in \mathbb{R}$ and $i=0,1$. Assume that there exists a vector $\hat{\mathbf{y}} \in \mathbb{C}^{l}$ such that $\Phi_{1}(\hat{\mathbf{y}})<0$. Then the following conditions are equivalent:

S1: $\Phi_{0}(\mathbf{y}) \geq 0$ for all $\mathbf{y} \in \mathbb{C}^{l}$ such that $\Phi_{1}(\mathbf{y}) \leq 0$.

S2: There exists $\lambda \geq 0$ such that the following LMI is feasible:

$$
\left[\begin{array}{ll}
\mathbf{A}_{0} & \mathbf{b}_{0} \\
\mathbf{b}_{0}^{H} & c_{0}
\end{array}\right]+\lambda\left[\begin{array}{cc}
\mathbf{A}_{1} & \mathbf{b}_{1} \\
\mathbf{b}_{1}^{H} & c_{1}
\end{array}\right] \succeq 0 .
$$

Consider the first constraint in Problem (15). It can be re-written as

$$
\begin{gathered}
\mathbf{e}_{n n}^{H} \mathbf{W}_{n} \mathbf{e}_{n n}+2 \operatorname{Re}\left(\left(\mathbf{W}_{n} \hat{\mathbf{g}}_{n n}\right)^{H} \mathbf{e}_{n n}\right)+\hat{\mathbf{g}}_{n n}^{H} \mathbf{W}_{n} \hat{\mathbf{g}}_{n n} \\
\quad-\gamma\left(\sum_{\substack{m=1 \\
m \neq n}}^{N} s_{m n}+\sigma_{n}^{2}\right) \geq 0, \quad n \in \mathcal{N}
\end{gathered}
$$

for all $\mathbf{e}_{n n} \in \mathbb{C}^{n_{t}}$ such that

$$
\mathbf{e}_{n n}^{H} \mathbf{Q}_{n n} \mathbf{e}_{n n}-1 \leq 0, \quad n \in \mathcal{N}
$$

should be satisfied. The existence of an $\mathbf{e}_{n n}$ for which (17) holds strictly is obvious (e.g., $\mathbf{e}_{n n}=\mathbf{0}$ ). Hence, we can regard the left-hand sides of (16) and (17) as $\Phi_{0}\left(\mathbf{e}_{n n}\right)$ and $\Phi_{1}\left(\mathbf{e}_{n n}\right)$ in S-lemma. Then, according to S-lemma, the inequality (16) is satisfied for all channel errors $\mathbf{e}_{n n}$ 
that satisfy (17) if there exists $\mu_{n n} \geq 0$ such that the condition

$$
\begin{aligned}
\boldsymbol{\Delta}_{n n} \triangleq & {\left[\begin{array}{cc}
\mathbf{W}_{n} & \mathbf{W}_{n} \hat{\mathbf{g}}_{n n} \\
\hat{\mathbf{g}}_{n n}^{H} \mathbf{W}_{n} & \hat{\mathbf{g}}_{n n}^{H} \mathbf{W}_{n} \hat{\mathbf{g}}_{n n}-\gamma\left(\sum_{\substack{m=1 \\
m \neq n}}^{N} s_{m n}+\sigma_{n}^{2}\right)
\end{array}\right] } \\
& +\mu_{n n}\left[\begin{array}{cc}
\mathbf{Q}_{n n} & 0 \\
0 & -1
\end{array}\right] \succeq 0, \quad n \in \mathcal{N}
\end{aligned}
$$

is satisfied.

Following the same procedure, it follows that the inequality (15c) is satisfied for all channel errors $\mathbf{e}_{m n} \in$ $\mathcal{E}_{m n}$ if there exists $\mu_{m n} \geq 0$ such that

$$
\begin{aligned}
\boldsymbol{\Phi}_{m n} \triangleq & {\left[\begin{array}{cc}
-\mathbf{W}_{m} & -\mathbf{W}_{m} \hat{\mathbf{g}}_{m n} \\
-\hat{\mathbf{g}}_{m n}^{H} \mathbf{W}_{m} & s_{m n}-\hat{\mathbf{g}}_{m n}^{H} \mathbf{W}_{m} \hat{\mathbf{g}}_{m n}
\end{array}\right] } \\
& +\mu_{m n}\left[\begin{array}{cc}
\mathbf{Q}_{m n} & 0 \\
0 & -1
\end{array}\right] \succeq 0, \quad m \in \mathcal{N} \backslash\{n\}, n \in \mathcal{N}
\end{aligned}
$$

is satisfied. Similarly, the inequality (15e) is satisfied for all channel errors $\tilde{\mathbf{e}}_{n k} \in \tilde{\mathcal{E}}_{m n}$ if there exists $v_{n k} \geq 0$ such that

$$
\begin{aligned}
\boldsymbol{\Theta}_{n k} \triangleq & {\left[\begin{array}{cc}
-\mathbf{W}_{n} & -\mathbf{W}_{n} \hat{\mathbf{h}}_{n k} \\
-\hat{\mathbf{h}}_{n k}^{H} \mathbf{W}_{n} & I_{n k}-\hat{\mathbf{h}}_{n k}^{H} \mathbf{W}_{n} \hat{\mathbf{h}}_{n k}
\end{array}\right] } \\
& +v_{n k}\left[\begin{array}{cc}
\tilde{\mathbf{Q}}_{n k} & 0 \\
0 & -1
\end{array}\right] \succeq 0, \quad n \in \mathcal{N}, k \in \mathcal{K}
\end{aligned}
$$

is satisfied.

Thus, we can rewrite Problem (15) equivalently as follows:

$$
\begin{array}{ll}
\text { maximize } & \gamma \\
\text { subject to } & \boldsymbol{\Delta}_{n n} \geq 0, \quad n \in \mathcal{N} \\
& \boldsymbol{\Phi}_{m n} \geq 0, \quad m \in \mathcal{N} \backslash\{n\}, n \in \mathcal{N} \\
& \boldsymbol{\Theta}_{n k} \geq 0, \quad n \in \mathcal{N}, k \in \mathcal{K} \\
& \sum_{n=1}^{N} I_{n k} \leq I_{\text {th }}, \quad k \in \mathcal{K} \\
& \mu_{m n} \geq 0, \quad n, m \in \mathcal{N} \\
& v_{n k} \geq 0, \quad n \in \mathcal{N}, k \in \mathcal{K} \\
& \mathbf{W}_{n} \geq 0, \quad n \in \mathcal{N} \\
& \operatorname{Trace}\left(\mathbf{W}_{n}\right) \leq P_{\max }, \quad n \in \mathcal{N} \\
& \operatorname{Rank}\left(\mathbf{W}_{n}\right)=1, \quad n \in \mathcal{N},
\end{array}
$$

where the optimization variables are $\gamma$ and $\mathbf{W}_{n}, \mu_{m n}, v_{n k}, s_{m n}, I_{n k}$ for $n, m \in \mathcal{N}, k \in \mathcal{K}$. Note that if the rank constraints are neglected, Problem (21) can be easily solved using the bisection method (see p. 146 in [31]) which provides the optimal solution for Problem (21).
Specifically, for a fixed $\gamma$, the feasibility can be checked by solving the problem

$$
\begin{array}{ll}
P_{0}(\gamma): \text { find } & \left\{\mathbf{W}_{n}, \mu_{m n}, v_{n k}, s_{m n}, I_{n k}\right\}_{n, m \in \mathcal{N}, k \in \mathcal{K}} \\
\text { subject to } & \boldsymbol{\Delta}_{n n}(\gamma) \geq 0, \quad n \in \mathcal{N} \\
& \boldsymbol{\Phi}_{m n} \geq 0, \quad m \in \mathcal{N} \backslash\{n\}, n \in \mathcal{N} \\
& \boldsymbol{\Theta}_{n k} \geq 0, \quad n \in \mathcal{N}, k \in \mathcal{K} \\
& \sum_{n=1}^{N} I_{n k} \leq I_{\text {th }}, \quad k \in \mathcal{K} \\
& \mu_{m n} \geq 0, \quad n, m \in \mathcal{N} \\
& v_{n k} \geq 0, \quad n \in \mathcal{N}, k \in \mathcal{K} \\
& \mathbf{W}_{n} \geq 0, \quad n \in \mathcal{N} \\
& \text { Trace }\left(\mathbf{W}_{n}\right) \leq P_{\max }, \quad n \in \mathcal{N},
\end{array}
$$

where the optimization variables are $\mathbf{W}_{n}, \mu_{m n}, v_{n k}, s_{m n}, I_{n k}$ for all $n, m \in \mathcal{N}, k \in \mathcal{K}$, using a standard SDP solver and the optimal solution is the maximum value of $\gamma$ for which Problem (22) is feasible.

However, it turns out that we can employ a simple trick to handle also the rank constraint of Problem (21). The procedure is based on replacing the dummy objective function in Problem (22) with the sum power minimization for the secondary network. Then the problem used to check the feasibility can be written as

$$
\begin{array}{lll}
P_{1}(\gamma): & \text { minimize } & \sum_{n=1}^{N} \text { Trace }\left(\mathbf{W}_{n}\right) \\
& \text { subject to } & \text { constraints of } P_{0}(\gamma)
\end{array}
$$

where the optimization variables are $\mathbf{W}_{n}, \mu_{m n}, v_{n k}, s_{m n}, I_{n k}$ for all $n, m \in \mathcal{N}, k \in \mathcal{K}$. Clearly, Problem (22) is feasible if and only if Problem (23) is feasible (because they have the same set of constraints). Furthermore, the following proposition ensures that Problem (23) returns always a set of rank one matrices $\mathbf{W}_{n}$.

Proposition 1. If Problem (23) is feasible (for a given $\gamma$ ), then the optimal matrices $\mathbf{W}_{n}^{\star}$ are always rank one, i.e., $\mathbf{W}_{n}^{\star}=\mathbf{w}_{n}^{\star} \mathbf{w}_{n}^{\star H}$ for all $n \in \mathcal{N}$.

\section{Proof. The proof is presented in Appendix 1.}

Note that for a given feasible value of $\gamma$, Problem (22) can also have higher rank solutions but Problem (23) has only rank one solutions. The optimal beamforming vectors that maximize the minimum SINR can be found directly by eigen-decomposition of $\mathbf{W}_{n}^{\star}$ for all $n \in \mathcal{N}$. This implies that the global optimal solution of the original Problem (11) is obtained.

Following iterative algorithm can be used to design the optimal robust beamforming vectors for an underlay CRN that maximize the minimum SINR of the cognitive users within a pre-defined accuracy $\epsilon>0$. 
In the bisection method, exactly $\left\lceil\log _{2}\left(\left(\gamma_{\text {upp }}-\gamma_{\text {low }}\right) / \epsilon\right)\right\rceil$ iterations are required before the algorithm terminates (see p. 146 in [31]), where $\gamma_{\text {low }}$ and $\gamma_{\text {upp }}$ are the initial lower and upper bounds for the optimal value. Hence, a good initialization can reduce the number of iterations required in the algorithm which leads to lower resource consumption. Therefore, in practice, it is highly beneficial to have good initial lower and upper bounds which are simple to obtain. Next, we describe an efficient method for finding lower and upper bounds (i.e., $\gamma_{\text {low }}$ and $\gamma_{\text {upp }}$ ) to initialize the above algorithm.

\subsection{Initial lower bound}

When a constraint in a maximization problem is modified (perturbed) in such a way that it gets tightened, the optimal value of the perturbed problem is always a lower bound for the optimal value of the original problem (see pp. 249-251 in [31]). We use this fact to compute an efficient initial lower bound, $\gamma_{\text {low }}$, for Algorithm 1 .

$\overline{\text { Algorithm } 1 \text { Robust cognitive beamforming via bisection }}$ method

given network parameters

$\left\{\hat{\mathbf{g}}_{m n}, \hat{\mathbf{h}}_{n k}, \mathbf{Q}_{m n}, \tilde{\mathbf{Q}}_{n k}, \sigma_{n}\right\}_{n, m \in \mathcal{N}, k \in \mathcal{K}}, I_{\text {th }}, P_{\max }$. tolerance $\epsilon>0$, initial lower and upper bounds for the optimal value $\gamma_{\text {low }}$ and $\gamma_{\text {upp }}$.

repeat

1. $\gamma:=\left(\gamma_{\text {low }}+\gamma_{\text {upp }}\right) / 2$.

2. Solve the convex feasibility Problem $P_{1}(\gamma)$.

3. if $P_{1}(\gamma)$ is feasible, $\gamma_{\text {low }}:=\gamma ;$ else $\gamma_{\text {upp }}:=\gamma$. until $\gamma_{\text {upp }}-\gamma_{\text {low }} \leq \epsilon$.

outputs $\gamma^{\star}$ and $\left\{\mathbf{w}_{n}^{\star}\left(\mathbf{w}_{n}^{\star}\right)^{H}=\mathbf{W}_{n}^{\star}\right\}_{n \in \mathcal{N}}$.

Consider the first constraint of Problem (15),

$$
\begin{gathered}
\left(\hat{\mathbf{g}}_{n n}+\mathbf{e}_{n n}\right)^{H} \mathbf{w}_{n} \mathbf{w}_{n}^{H}\left(\hat{\mathbf{g}}_{n n}+\mathbf{e}_{n n}\right) \geq \gamma\left(\sum_{\substack{m=1 \\
m \neq n}}^{N} s_{m n}+\sigma_{n}^{2}\right), \\
n \in \mathcal{N} .
\end{gathered}
$$

We introduce a new variable $t$, that should satisfy the inequality

$$
t \geq \sum_{\substack{m=1 \\ m \neq n}}^{N} s_{m n}+\sigma_{n}^{2}, \quad n \in \mathcal{N} .
$$

Now, using (24) and (25), the constraint (15b) can be tightened as follows:

$$
\begin{aligned}
& \left(\hat{\mathbf{g}}_{n n}+\mathbf{e}_{n n}\right)^{H} \mathbf{w}_{n} \mathbf{w}_{n}^{H}\left(\hat{\mathbf{g}}_{n n}+\mathbf{e}_{n n}\right) \geq \gamma t, \quad n \in \mathcal{N}, \\
& \sum_{\substack{m=1 \\
m \neq n}}^{N} s_{m n}+\sigma_{n}^{2} \leq t, \quad n \in \mathcal{N} .
\end{aligned}
$$

We define a new set of variables $x=\frac{1}{t}$ and $u_{m n}=\frac{s_{m n}}{t}$, $y_{n k}=\frac{I_{n k}}{t}, \mathbf{V}_{n}=\frac{\mathbf{w}_{n} \mathbf{w}_{n}^{H}}{t}$ for $n \in \mathcal{N}, k \in \mathcal{K}$. Then, the perturbed problem can be written as

$$
\begin{array}{ll}
\operatorname{maximize} & \gamma \\
\text { subject to } & \left(\hat{\mathbf{g}}_{n n}+\mathbf{e}_{n n}\right)^{H} \mathbf{V}_{n}\left(\hat{\mathbf{g}}_{n n}+\mathbf{e}_{n n}\right) \geq \gamma, \\
& \forall \mathbf{e}_{n n} \in \mathcal{E}_{n n}, n \in \mathcal{N} \\
& \sum_{\substack{m=1 \\
m \neq n}}^{N} u_{m n}+\sigma_{n}^{2} x \leq 1, \quad n \in \mathcal{N} \\
& \left(\hat{\mathbf{g}}_{m n}+\mathbf{e}_{m n}\right)^{H} \mathbf{V}_{m}\left(\hat{\mathbf{g}}_{m n}+\mathbf{e}_{m n}\right) \leq u_{m n}, \\
& \forall \mathbf{e}_{m n} \in \mathcal{E}_{m n}, m \in \mathcal{N} \backslash\{n\}, n \in \mathcal{N} \\
& \sum_{n=1}^{N} y_{n k} \leq I_{\mathrm{th}}, \quad k \in \mathcal{K} \\
& \left(\hat{\mathbf{h}}_{n k}+\tilde{\mathbf{e}}_{n k}\right)^{H} \mathbf{V}_{n}\left(\hat{\mathbf{h}}_{n k}+\tilde{\mathbf{e}}_{n k}\right) \leq I_{n k}, \\
& \forall \tilde{\mathbf{e}}_{n k} \in \tilde{\mathcal{E}}_{n k}, n \in \mathcal{N}, k \in \mathcal{K} \\
& \operatorname{Trace}\left(\mathbf{V}_{n}\right) \leq P_{\max } x, \quad n \in \mathcal{N} \\
& \operatorname{Rank}\left(\mathbf{V}_{n}\right)=1, \quad n \in \mathcal{N},
\end{array}
$$

where the optimization variables are $\gamma, x$ and $\mathbf{V}_{n}, \mathbf{e}_{m n}, \tilde{\mathbf{e}}_{n k}, u_{m n}, y_{n k}$ for all $n, m \in \mathcal{N}, k \in \mathcal{K}$.

Following the same method as in Section 4.1, using S-lemma to recast the quadratic constraints as LMIs, Problem (28) can be reformulated as follows:

$$
\begin{aligned}
\operatorname{maximize} & \gamma \\
\text { subject to } & \check{\boldsymbol{\Delta}}_{n n} \succeq 0, \quad n \in \mathcal{N} \\
& \check{\boldsymbol{\Phi}}_{m n} \geq 0, \quad m \in \mathcal{N} \backslash\{n\}, n \in \mathcal{N} \\
& \check{\boldsymbol{\Theta}}_{n k} \succeq 0, \quad n \in \mathcal{N}, k \in \mathcal{K} \\
& \sum_{\substack{m=1 \\
m \neq n}}^{N} u_{m n}+\sigma_{n}^{2} x \leq 1, \quad n \in \mathcal{N} \\
& \sum_{n=1}^{N} y_{n k} \leq I_{\mathrm{th}} x, \quad k \in \mathcal{K} \\
& \check{\mu}_{m n} \geq 0, \quad n, m \in \mathcal{N} \\
& \check{v}_{n k} \geq 0, \quad n \in \mathcal{N}, k \in \mathcal{K} \\
& \mathbf{V}_{n} \geq 0, \quad n \in \mathcal{N} \\
& \operatorname{Trace}\left(\mathbf{V}_{n}\right) \leq P_{\max } x, \quad n \in \mathcal{N} \\
& \operatorname{Rank}\left(\mathbf{V}_{n}\right)=1, \quad n \in \mathcal{N},
\end{aligned}
$$


where

$$
\begin{aligned}
& \check{\boldsymbol{\Delta}}_{n n}=\left[\begin{array}{cc}
\mathbf{V}_{n} & \mathbf{V}_{n} \hat{\mathbf{g}}_{n n} \\
\hat{\mathbf{g}}_{n n}^{H} \mathbf{V}_{n} & \hat{\mathbf{g}}_{n n}^{H} \mathbf{V}_{n} \hat{\mathbf{g}}_{n n}-\gamma
\end{array}\right]+\check{\mu}_{n n}\left[\begin{array}{cc}
\mathbf{Q}_{n n} & 0 \\
0 & -1
\end{array}\right] \\
& \check{\boldsymbol{\Phi}}_{m n}=\left[\begin{array}{cc}
-\mathbf{V}_{m} & -\mathbf{V}_{m} \hat{\mathbf{g}}_{m n} \\
-\hat{\mathbf{g}}_{m n}^{H} \mathbf{W}_{m} & u_{m n}-\hat{\mathbf{g}}_{m n}^{H} \mathbf{V}_{n} \hat{\mathbf{g}}_{m n}
\end{array}\right]+\check{\mu}_{m n}\left[\begin{array}{cc}
\mathbf{Q}_{m n} & 0 \\
0 & -1
\end{array}\right]
\end{aligned}
$$

$$
\check{\boldsymbol{\Theta}}_{n k}=\left[\begin{array}{cc}
-\mathbf{V}_{n} & -\mathbf{V}_{n} \hat{\mathbf{h}}_{n k} \\
-\hat{\mathbf{h}}_{n k}^{H} \mathbf{V}_{n} & y_{n k}-\hat{\mathbf{h}}_{n k}^{H} \mathbf{V}_{n} \hat{\mathbf{h}}_{n k}
\end{array}\right]+\check{v}_{n k}\left[\begin{array}{cc}
\tilde{\mathbf{Q}}_{n k} & 0 \\
0 & -1
\end{array}\right]
$$

and the optimization variables are $\gamma, x$ and $\mathbf{V}_{n}, \check{\mu}_{m n}, \check{v}_{n k}, u_{m n}, y_{n k}$ for all $n, m \in \mathcal{N}, k \in \mathcal{K}$. Note that the non-convex product terms $\gamma s_{m n}$ (in Problem (21)) are not present in Problem (29). Hence, without the rank constraints the Problem (29) is convex (even when $\gamma$ is considered as a variable). Therefore, the relaxed problem can be efficiently solved using SDP solvers such as SeDuMi [36] or SDPT3 [37].

Let us denote the optimal matrices $\mathbf{V}_{n}$ for Problem (29) by $\mathbf{V}_{n}^{\star}$ for all $n \in \mathcal{N}$. Then a rank-one feasible solution for Problem (29) can be achieved by eigenvalue decomposition as follows:

$$
\mathbf{V}_{n}^{\mathrm{ei}}=\lambda_{\max } \mathbf{y}_{\max } \mathbf{y}_{\max }^{H},
$$

where $\lambda_{\max }$ is the maximum eigenvalue and $\mathbf{y}_{\max }$ represents the corresponding eigenvector of matrix $\mathbf{V}_{n}^{\star}$. The feasibility of $\mathbf{V}_{n}^{\mathrm{ei}}$ is proved in Appendix 2 . Since the matrices $\mathbf{V}_{n}^{\text {ei }}$ are feasible for the perturbed Problem (29), the corresponding objective value obtained by solving Problem (29) is a lower bound for Problem (15).

\subsection{Initial upper bound}

An upper bound for a maximization problem can be found by relaxing a constraint of the original problem. Specifically, here we relax the first constraint,

$$
\gamma \leq \frac{\left|\left(\hat{\mathbf{g}}_{n n}+\mathbf{e}_{n n}\right)^{H} \mathbf{w}_{n}\right|^{2}}{\sum_{\substack{m=1 \\ m \neq n}}^{N}\left|\left(\hat{\mathbf{g}}_{m n}+\mathbf{e}_{m n}\right)^{H} \mathbf{w}_{m}\right|^{2}+\sigma_{n}^{2}}, \quad n \in \mathcal{N},
$$

in Problem (12). Since the interference from the $m$ th secondary TX to the $n$th secondary $\mathrm{RX}$ is a non-negative entity, i.e., $\left|\left(\hat{\mathbf{g}}_{m n}+\mathbf{e}_{m n}\right)^{H} \mathbf{w}_{m}\right|^{2} \geq 0$, the constraint (34) can be relaxed as

$$
\gamma \leq \frac{\left|\left(\hat{\mathbf{g}}_{n n}+\mathbf{e}_{n n}\right)^{H} \mathbf{w}_{n}\right|^{2}}{\sigma_{n}^{2}}, \quad n \in \mathcal{N} .
$$

Now the perturbed Problem (12) with the relaxed constraint can be written as

maximize $\gamma$

$$
\begin{array}{ll}
\text { subject to } \quad & \gamma \leq \frac{\left|\left(\hat{\mathbf{g}}_{n n}+\mathbf{e}_{n n}\right)^{H} \mathbf{w}_{n}\right|^{2}}{\sigma_{n}^{2}}, \quad \forall \mathbf{e}_{n n} \in \mathcal{E}_{n n}, n \in \mathcal{N} \\
& \sum_{n=1}^{N}\left|\left(\hat{\mathbf{h}}_{n k}+\tilde{\mathbf{e}}_{n k}\right)^{H} \mathbf{w}_{n}\right|^{2} \leq I_{\mathrm{th}}, \\
& \forall \tilde{\mathbf{e}}_{n k} \in \tilde{\mathcal{E}}_{n k}, n \in \mathcal{N}, k \in \mathcal{K} \\
& \left\|\mathbf{w}_{n}\right\|_{2}^{2} \leq P_{\max }, \quad n \in \mathcal{N},
\end{array}
$$

where the optimization variables are $\gamma$ and $\mathbf{w}_{n}, \mathbf{e}_{n n}, \tilde{\mathbf{e}}_{n k}$ for $n \in \mathcal{N}, k \in \mathcal{K}$.

Following a similar approach as in sections 3.2 and 4.1, by introducing variables $\mathbf{W}_{n}=\mathbf{w}_{n} \mathbf{w}_{n}^{H}, I_{n k}=\mid\left(\hat{\mathbf{h}}_{n k}+\right.$ $\left.\tilde{\mathbf{e}}_{n k}\right)\left.^{H} \mathbf{w}_{n}\right|^{2}$ and using the S-lemma to recast the quadratic constraints, Problem (36) can be reformulated as

$$
\begin{array}{ll}
\operatorname{maximize} & \gamma \\
\text { subject to } & \overline{\boldsymbol{\Delta}}_{n n} \geq 0, \quad n \in \mathcal{N}, \\
& \overline{\boldsymbol{\Theta}}_{n k} \geq 0, \quad n \in \mathcal{N}, k \in \mathcal{K} \\
& \sum_{n=1}^{N} I_{n k} \leq I_{\mathrm{th}}, \quad k \in \mathcal{K} \\
& \bar{\mu}_{n n} \geq 0, \quad n \in \mathcal{N} \\
& \bar{v}_{n k} \geq 0, \quad n \in \mathcal{N}, k \in \mathcal{K} \\
& \mathbf{W}_{n} \geq 0, \quad n \in \mathcal{N} \\
& \operatorname{Trace}\left(\mathbf{W}_{n}\right) \leq P_{\max }, \quad n \in \mathcal{N} \\
& \operatorname{Rank}\left(\mathbf{W}_{n}\right)=1, \quad n \in \mathcal{N},
\end{array}
$$

where

$$
\overline{\boldsymbol{\Delta}}_{n n}=\left[\begin{array}{cc}
\mathbf{W}_{n} & \mathbf{W}_{n} \hat{\mathbf{g}}_{n n} \\
\hat{\mathbf{g}}_{n n}^{H} \mathbf{W}_{n} & \hat{\mathbf{g}}_{n n}^{H} \mathbf{W}_{n} \hat{\mathbf{g}}_{n n}-\gamma \sigma_{n}^{2}
\end{array}\right]+\bar{\mu}_{n n}\left[\begin{array}{cc}
\mathbf{Q}_{n n} & 0 \\
0 & -1
\end{array}\right]
$$

$$
\overline{\boldsymbol{\Theta}}_{n k}=\left[\begin{array}{cc}
-\mathbf{W}_{n} & -\mathbf{W}_{n} \hat{\mathbf{h}}_{n k} \\
-\hat{\mathbf{h}}_{n k}^{H} \mathbf{W}_{n} & I_{n k}-\hat{\mathbf{h}}_{n k}^{H} \mathbf{W}_{n} \hat{\mathbf{h}}_{n k}
\end{array}\right]+\bar{v}_{n k}\left[\begin{array}{cc}
\tilde{\mathbf{Q}}_{n k} & 0 \\
0 & -1
\end{array}\right]
$$

and the optimization variables are $\gamma$ and $\mathbf{W}_{n}, \bar{\mu}_{n n}, \bar{v}_{n k}, I_{n k}$ for all $n \in \mathcal{N}, k \in \mathcal{K}$. Without the rank constraints, Problem (37) can be efficiently solved using existing SDP solvers. Note that the non-convex product terms $\gamma s_{m n}$ (in Problem (21)) are not present in Problem (37). Hence, without the rank constraints, Problem (37) is convex (even when we consider $\gamma$ as a variable). Further, the optimal solution of Problem (37) gives an upper bound for Problem (15). It should be mentioned that we solve a relaxed 
version of Problem (37). Hence, the optimal solution of the relaxed problem also provides an upper bound for Problem (15).

\subsection{Extension to a more general objective function}

Consider the resource allocation problem where the CRN is required to optimize the transmit beamforming vectors to maximize the worst weighted SINR or, more generally the worst among a set of increasing functions of each SINR. Let $f_{n}$ be an increasing function of $\operatorname{SINR}_{n}$. Since transmit power and primary interference constraints are inherited in underlay CRNs, for a bounded uncertainty in the channel errors, this problem can be mathematically expressed $^{\mathrm{d}}$ as

$$
\begin{array}{ll}
\operatorname{maximize} & \min _{n=1, \ldots, N} \inf _{\mathbf{e}_{m n} \in \mathcal{E}_{m n}, m \in \mathcal{N} f_{n}\left(\operatorname{SINR}_{n}\right)} \\
\text { subject to } & \sup _{\tilde{\mathbf{e}}_{n k} \in \tilde{\mathcal{E}}_{n k}, n \in \mathcal{N}} I_{k} \leq I_{\mathrm{th}}, \quad k \in \mathcal{K} \\
& \left\|\mathbf{w}_{n}\right\|_{2}^{2} \leq P_{\max } \quad n \in \mathcal{N}
\end{array}
$$

where the optimization variables are $\mathbf{w}_{n}, \mathbf{e}_{m n}, \tilde{\mathbf{e}}_{n k}$ for $n, m \in \mathcal{N}, k \in \mathcal{K}$.

The function $f_{n}$ is an increasing function of $\operatorname{SINR}_{n}$; hence,

$$
\inf _{\mathbf{e}_{m n} \in \mathcal{E}_{m n}, m \in \mathcal{N}} f_{n}\left(\operatorname{SINR}_{n}\right)=f_{n}\left(\inf _{\mathbf{e}_{m n} \in \mathcal{E}_{m n}, m \in \mathcal{N}} \operatorname{SINR}_{n}\right) .
$$

Therefore, the optimization Problem (40) can be equivalently written in the hypograph form (see p. 134 in [31]) as

maximize $\gamma$

$$
\begin{gathered}
\text { subject to } f_{n}^{-1}(\gamma) \leq \frac{\left|\left(\hat{\mathbf{g}}_{n n}+\mathbf{e}_{n n}\right)^{H} \mathbf{w}_{n}\right|^{2}}{\sum_{\substack{m=1 \\
m \neq n \\
\forall \neq n}}^{N}\left|\left(\hat{\mathbf{g}}_{m n}+\mathbf{e}_{m n}\right)^{H} \mathbf{w}_{m}\right|^{2}+\sigma_{n}^{2}}, \\
\sum_{n=1}^{N}\left|\left(\hat{\mathbf{h}}_{n k}+\tilde{\mathbf{e}}_{n k}\right)^{H} \mathbf{w}_{n}\right|^{2} \leq I_{\mathrm{th}}, \forall \tilde{\mathbf{e}}_{n k} \in \tilde{\mathcal{E}}_{n k}, \\
k \in \mathcal{K}\left\|\mathbf{w}_{n}\right\|_{2}^{2} \leq P_{\max }, \quad n \in \mathcal{N},
\end{gathered}
$$

where the optimization variables are $\gamma$ and $\mathbf{w}_{n}, \mathbf{e}_{m n}, \tilde{\mathbf{e}}_{n k}$ for $n, m \in \mathcal{N}, k \in \mathcal{K}$. Problem (41) has the similar format as Problem (12), the only difference is that $\gamma$ in the first constraint of Problem (12) should be replaced by the evaluated function value $f_{n}^{-1}(\gamma)$ in Problem (41). Hence, following the similar steps as in sections 3.2 and 4 , the Problem $P_{1}(\gamma)$ can be modified according to the new constraint. Then Problem (40) can be solved optimally by directly applying Algorithm 1 with the modified Problem $P_{1}(\gamma)$ to check the feasibility.

\section{Results and discussion}

Numerical simulations are performed to validate and assess the performance of the proposed beamforming scheme. We consider a network model where two secondary TX-RX pairs $(N=2)$ coexist in a primary network with two primary RXs $(K=2)$. We assume that each secondary TX is equipped with four antennas $\left(n_{t}=4\right)$. Further, all complex entries of the estimated channel vectors are independent and identically distributed (i.i.d) according to $\mathcal{C N}(0,1)$. We assume that $\mathbf{Q}_{m n}=\tilde{\mathbf{Q}}_{m k}=\left(1 / \xi^{2}\right) \mathbf{I}_{n_{t}}$ for all $n, m \in \mathcal{N}, k \in \mathcal{K}$. Thus, an estimation error can take any value inside a ball with radius $\xi$. We use a truncated normal distribution $\mathcal{T C N}\left(0, \xi^{2} / 9 n_{t},-\xi / \sqrt{2 n_{t}},+\xi / \sqrt{2 n_{t}}\right)$ (see [33], Chap. 4 in [34]) to generate each complex entry of the estimation errors. Additionally, we assume that $\sigma_{n}^{2}=\sigma^{2}=1$ for all $n \in \mathcal{N}$ and the maximum available transmit power of a secondary TX is $P_{\max }=10 \sigma^{2}$. To maintain the QoS requirements for the primary network, we limit the maximum allowable interference power from the secondary network in the order of the received noise power, i.e., $I_{\text {th }}=\sigma^{2}(=1)$.

We present results for both robust and non-robust beamforming designs for comparison. The robust beamforming vectors are acquired directly following Algorithm 1 with tolerance $\epsilon=10^{-2}$. For the non-robust case, the beamforming vectors are obtained based only on the estimated channels and ignoring the uncertainty. We follow the Algorithm 1 except that at step 2, we replace Problem (23) by the following one:

$\operatorname{minimize} \sum_{n=1}^{N} \operatorname{Trace}\left(\mathbf{W}_{n}\right)$

$$
\begin{aligned}
\text { subject to } & \hat{\mathbf{g}}_{n n}^{H} \mathbf{W}_{n} \hat{\mathbf{g}}_{n n} \geq \gamma\left(\sum_{\substack{m=1 \\
m \neq n}}^{N} \hat{\mathbf{g}}_{m n}^{H} \mathbf{W}_{m} \hat{\mathbf{g}}_{m n}+\sigma_{n}^{2}\right), \\
& n \in \mathcal{N} \quad \sum_{n=1}^{N} \hat{\mathbf{h}}_{n k}^{H} \mathbf{W}_{n} \hat{\mathbf{h}}_{n k}-I_{\text {th }} \leq 0, \quad k \in \mathcal{K} \\
& \text { Trace }\left(\mathbf{W}_{n}\right)-P_{\max } \leq 0, \quad n \in \mathcal{N} \\
& \mathbf{W}_{n} \succeq 0, \quad n \in \mathcal{N},
\end{aligned}
$$

where the optimization variables are $\mathbf{W}_{n}$ for all $n \in \mathcal{N}$. The MATLAB toolbox CVX [38] is used to solve all the optimization problems, and there, the SDPs are solved using the SeDuMi solver.

Figure 2 displays the empirical cumulative density function (CDF) of the interference due to the secondary network at first primary RX for different values of $\xi$ (i.e., radius of the uncertainty ball). The empirical CDF is calculated over a set of 10,000 different channel errors inside the given uncertainty region for a fixed estimated channel realization. As expected, the non-robust design can not guarantee the maximum interference threshold even 


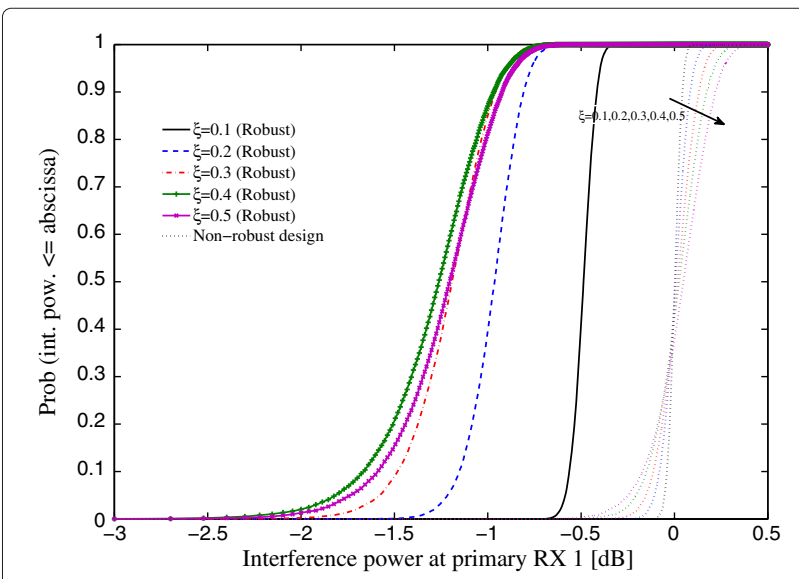

Figure $\mathbf{2} C D F$ of the interference due to the secondary network at a primary RX. Empirical CDF of the interference due to the secondary network at first primary RX for different values of $\xi$. A network with parameters $n_{t}=4, N=2$ and $K=2$ is considered. The interference threshold for the primary network is $1(0 \mathrm{~dB})$. The empirical CDF is calculated over different channel errors for a fixed estimated channel realization.

when the CSI uncertainties are relatively small. Specifically, with non-robust design, around 52\%, 55\%, 57\%, 59\% and $61 \%$ of the simulated channel errors exceed the interference threshold of the primary network $\left(I_{\text {th }}=1\right)$ for uncertainty ball radius of $0.1,0.2,0.3,0.4$ and 0.5 , respectively. On the other hand, for the proposed robust algorithm, the interference to the primary RX is always less than the threshold level ${ }^{\mathrm{e}}$, which guarantees the required QoS of the primary network.

In Figure 3, we plot the empirical CDF of the interference due to the secondary network at first primary $\mathrm{RX}$

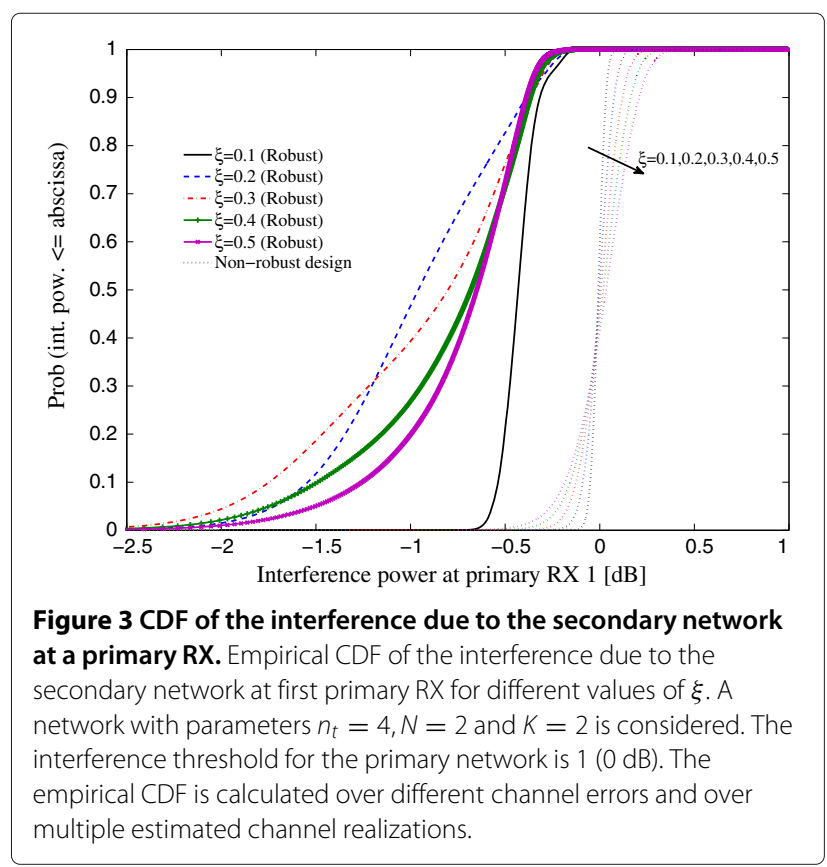

for different values of $\xi$ averaged over different estimated channel realizations. We generated 1,000 distinct estimated channel realizations and for each of them, 10,000 different channel errors are generated to calculate the empirical CDF. It can be seen that for more than half of the simulated cases, regardless the level of uncertainty, the non-robust solution exceeds the maximum interference threshold. On the other hand, the robust design guarantees the interference to the primary $\mathrm{RX}$ remain below the interference threshold $\left(I_{\text {th }}=1\right)$ for all simulated cases. Moreover, it can be observed from Figures 2 and 3 that the variation in interference power becomes large with $\xi$. When the uncertainty region is small (i.e., small $\xi$ ), the beamformer can be designed to direct relatively sharp nulls towards primary RXs for all possible channel values within the uncertainty region. As the radius $\xi$ increases, the range of possible channel values inside the uncertainty region increases as well and causes the nulls to become less focused. Hence, the interference power varies in a wider range for different channel errors inside the uncertainty region.

Figure 4 shows the empirical CDF of the received SINR at the second secondary RX for different values of $\xi$ (i.e., the radius of uncertainty balls). We plot the SINR at the second secondary RX since it gives the minimum SINR for the considered value of the channel estimate. As in Figure 2, the empirical CDF is taken over 10,000 possible channel errors inside the uncertainty region. When the uncertainty region is small, the beamformer can be designed to direct relatively sharp nulls towards the primary RXs for all possible channel values within the uncertainty region. Hence, higher transmit powers can be allocated to the sharp beams towards the secondary RXs resulting in higher SINR values for smaller $\xi$ values. The

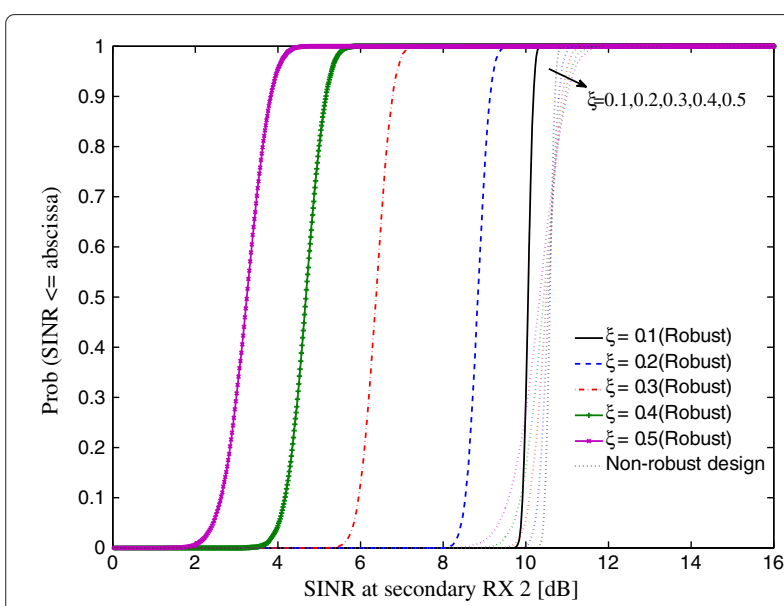

Figure $4 \mathrm{CDF}$ of the received SINR at a secondary RX. Empirical CDF of the received SINR at second secondary $R X$ for different values of $\xi$. A network with parameters $n_{t}=4, N=2$ and $K=2$ is considered. The empirical CDF is calculated over different channel errors for a fixed estimated channel realization. 
ability of the secondary TXs to focus sharp beams towards the corresponding secondary RXs while simultaneously directing nulls towards other secondary and primary RXs reduces as the channel knowledge decreases (i.e., when $\xi$ increases). Hence, the mean SINR reduces with the increase in the radius of uncertainty balls.

Figure 5 displays the empirical CDF of the received SINR at a specific secondary RX for different values of $\xi$, averaged over multiple estimated channels. As in Figure 3, we produced 1,000 different estimated channels, and for each of them, 10,000 different channel errors are generated to calculate the empirical CDF. We can observe that the SINR performance of the robust design with $\xi=0.1$ is similar to the SINR performance of the non-robust design which is not able to guarantee the interference threshold. Thus, in average, our robust design provides a similar SINR performance for the secondary network as the non-robust design without exceeding the interference threshold of the primary network. Further, we notice a reduction in SINR when the uncertainty region increases. This is mainly due to the fact that the transmitter is not able to use all the available transmit power when the uncertainty region is large. This effect can be clearly seen in the next figure.

Figure 6 presents the transmit power variation at a specific secondary TX with $\xi$ (i.e., the radius of uncertainty balls). This figure is drawn using the same setup as in Figures 3 and 5 . When the uncertainty region is small, since the nulls towards the primary RXs are sharp, higher transmit powers can be allocated to secondary TXs to achieve higher SINR values at secondary RXs. As $\xi$ decreases from 0.3 to 0 , we notice a rapid increase in the transmit power. On the other hand, when the uncertainty

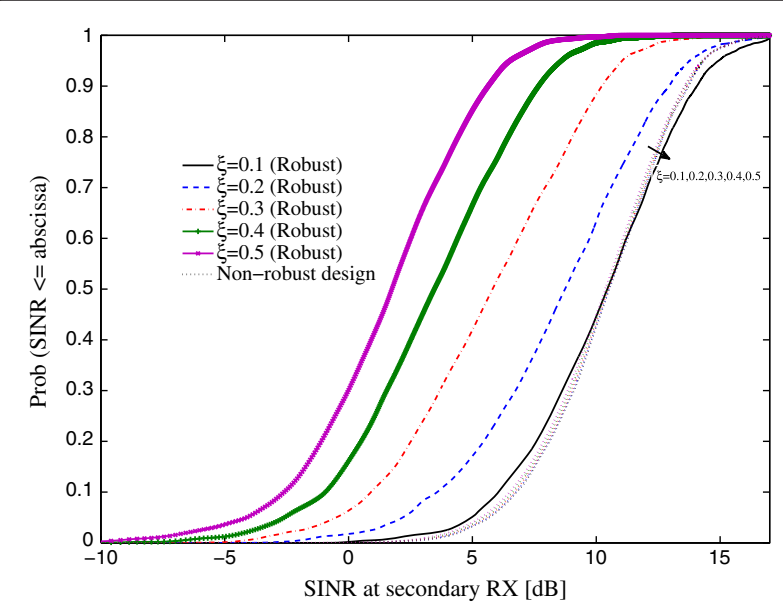

Figure 5 CDF of the received SINR at a secondary RX. Empirical CDF of the received SINR at a secondary RX for different values of $\xi$. A network with parameters $n_{t}=4, N=2$ and $K=2$ is considered. The empirical CDF is calculated over different channel errors and over multiple estimated channel realizations.

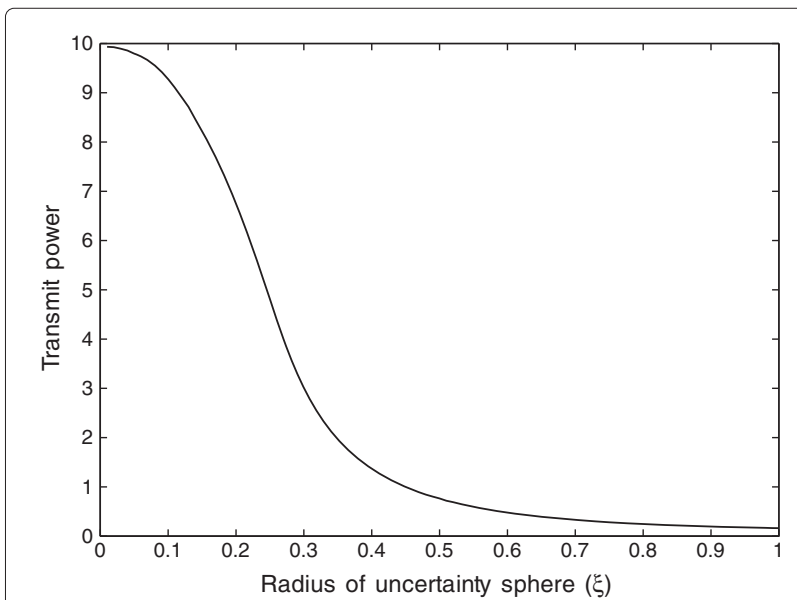

Figure 6 Transmit power variation at a secondary TX. The

transmit power variation at a secondary $T X$ with respect to the radius of the uncertainty balls ( $\xi$ ). A network with parameters $n_{t}=4, N=2$ and $K=2$ is considered. Transmit power is averaged over 1,000 estimated channel realizations.

region is large, the beamformer design cannot focus sharp nulls towards primary RXs for all possible channel values in the uncertainty region. Therefore, the transmitter has to decrease its transmit power to be able to guarantee the interference threshold for all possible channel values in the uncertainty region. Hence, for $\xi$ values greater than 0.5 , the transmit power has become small.

Next, we illustrate the gain pattern variation of the beamforming vectors designed from non-robust and robust designs for different uncertainty regions (i.e., different values of $\xi$ ). For illustration purposes, we consider a simple network model where a single secondary TX-RX pair $(N=1)$ coexist in a primary network with three primary RXs ( $K=3$; see Figure 7). Further, we use a simple line-of-sight model for the channels since the physical meaning of a beamforming pattern in a rich scattering environment is difficult to visualize with respect to the locations of the receivers. We model the estimated channel vector from the secondary TX to secondary RX as $\mathbf{g}_{11}=\left[g_{11}^{1}, \ldots, g_{11}^{i}, \ldots, g_{11}^{n_{t}}\right]^{T}$, where the channel from antenna element $i$ to secondary RX is given by [17]

$$
g_{11}^{i}=\frac{1}{\sqrt{n_{t}}} \exp \left(2 \pi j / \lambda\left(x_{i} \cos \theta+y_{i} \sin \theta\right)\right),
$$

where $\left(x_{i}, y_{i}\right)$ is the location of the $i$ th antenna element, $\theta$ is the direction of the RX with respect to the direction of the antenna array and $j=\sqrt{-1}$. Similarly, we model the estimated channel vector from the secondary TX to the $k$ th primary $\mathrm{RX}$ as $\mathbf{h}_{1 k}=\left[h_{1 k}^{1}, \ldots, h_{1 k}^{i}, \ldots, h_{1 k}^{n_{t}}\right]^{T}$, where $h_{1 k}^{i}=\frac{1}{\sqrt{n_{t}}} \exp \left(2 \pi j / \lambda\left(x_{i} \cos \theta+y_{i} \sin \theta\right)\right)$ gives the channel from antenna element $i$ to the $k$ th primary $\mathrm{RX}$. We assume that the secondary TX is equipped with $n_{t}=10$ antennas arranged in a linear array and spaced 


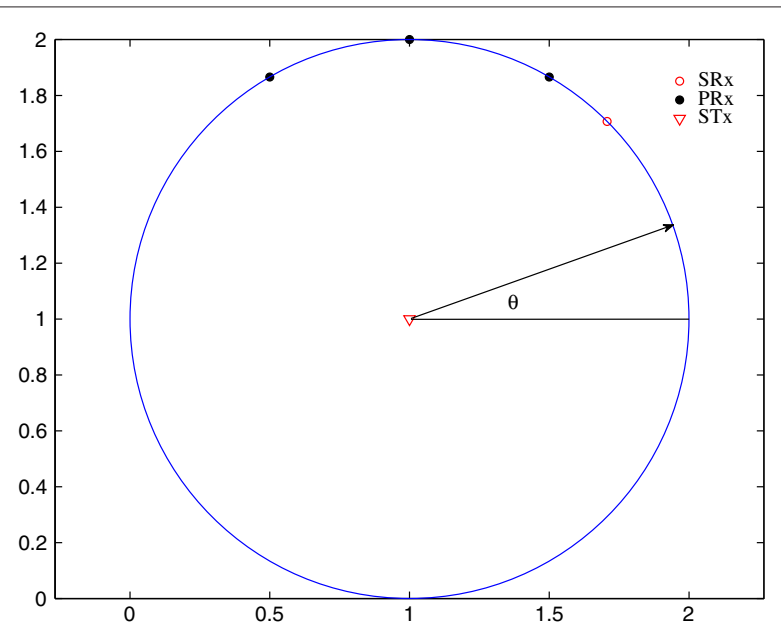

Figure 7 Network model for gain pattern comparison. Sample network. Secondary receiver at direction $\theta=45^{\circ}$ and primary receivers at directions $\theta=60^{\circ}, \theta=90^{\circ}$ and $\theta=120^{\circ}$. Network with parameters $n_{t}=10, N=1$ and $K=3$. Antennas lie in a linear array $\lambda / 2$ apart, centered at transmitter and along the direction $\theta=0^{\circ}$.

half-a-wavelength $(\lambda / 2)$ apart. Further, the antenna array is centered at the transmitter and lies along the direction $\theta=0^{\circ}$. The secondary RX is located at direction $\theta=45^{\circ}$ and the primary RXs are located at directions $\theta=60^{\circ}$, $\theta=90^{\circ}$ and $\theta=120^{\circ}$.

Figure 8 shows the gain patterns for the non-robust beamforming vector designed with estimated channels and robust beamforming vectors designed with Algorithm 1. As expected, the gain reduces with $\xi$, reducing the SINR at the secondary RX since the TX have to decrease its transmit power to be able to guarantee the interference threshold for primary RXs. Further, as

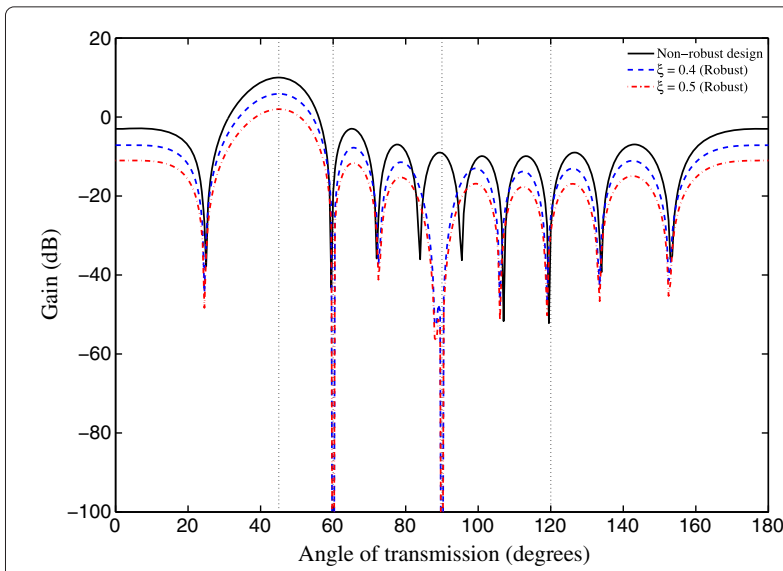

Figure 8 Gain pattern comparison. Gain pattern comparison for robust and non-robust beamforming designs. Network with parameters $n_{t}=10, N=1$ and $K=3$. Antennas lie in a linear array $\lambda / 2$ apart, centered at a transmitter and along the direction $\theta=0^{\circ}$. Vertical dotted lines represent the directional location of the receivers (secondary receiver $\theta=45^{\circ}$, primary receivers $\theta=60^{\circ}, 90^{\circ}, 120^{\circ}$ ). $\xi$ increases, we notice that the nulls around the angles at which the primary RXs are located become wider in order to protect the primary system ${ }^{\mathrm{f}}$. Therefore, Figure 8 validates the previous observations that as $\xi$ increases the transmit power decreases reducing the SINR at secondary RXs and nulls become wider around the angles at which the primary RXs are located to protect the primary system, explaining the trade-off between secondary performance and the protection for the primary system.

\section{Conclusion}

We have proposed a method to design the optimal transmit beamformer vectors for an underlay cognitive radio network, which maximizes the minimum received SINRs of the cognitive users subject to primary users' interference constraints. We have considered that the network controller has imperfect CSI knowledge in all relevant channels, and bounded ellipsoidal uncertainty model has been used to model the CSI errors. First, we have converted this highly untractable non-convex problem into a convex problem by means of semidefinite relaxation. Then, an iterative algorithm has been proposed based on the bisection method to solve the relaxed problem. Furthermore, we have provided a method to obtain the optimal solution for the original problem (i.e., non-relaxed problem) by introducing a properly chosen objective function into the iterative algorithm. Finally, upper and lower bounds for the optimal value of the considered problem have been proposed, which provides better initialization for the algorithm. The simulation results show that the performance of the primary network is guaranteed by the proposed algorithm for any channel error within the considered uncertainty region. Furthermore, results show that for smaller uncertainty regions, the SINR performance of the proposed robust design is similar to that of the non-robust design.

\section{Endnotes}

${ }^{\text {a }}$ We can apply the mathematical model of the proposed network to more general network scenarios. As an example, we can consider a multi-cell network where the transmitters are base stations (BSs) equipped with multiple antennas that communicate with the single antenna mobile stations (MSs) using time-divisionmultiple-access. A BS transmits signals to a single MS at a given time instant and has per-transmission power constraints. Further, the multi-cell network is subject to some interference temperature constraints.

${ }^{\mathrm{b}}$ We assume that the equivalent channel and the power of interference are perfectly known at each receiver.

${ }^{\mathrm{c}}$ Note that the infimum and the supremum in Problem (11) implies that the solution should satisfy for all channel errors inside the given uncertainty region. Therefore, we have written them equivalently as 
$\forall \mathbf{e}_{m n} \in \mathcal{E}_{m n}$ and $\forall \tilde{\mathbf{e}}_{n k} \in \tilde{\mathcal{E}}_{n k}$ in the first and second constraints of Problem (12), respectively. We can see that the variables $\gamma$ and $\mathbf{w}_{n}, \mathbf{e}_{m n}, \tilde{\mathbf{e}}_{n k}$ for $n, m \in \mathcal{N}, k \in \mathcal{K}$ are optimal for Problem (12) if and only if $\mathbf{w}_{n}, \mathbf{e}_{m n}, \tilde{\mathbf{e}}_{n k}$ for $n, m \in \mathcal{N}, k \in \mathcal{K}$ are optimal for Problem (11) and the first inequality constraint of Problem (12) holds with equality. Hence, the optimal solution of Problem (12) is equivalent to that of Problem (11).

${ }^{\mathrm{d}}$ Note that in the worst weighted SINR case the increasing functions $f_{n}\left(\operatorname{SINR}_{n}\right)=\zeta_{n} \operatorname{SINR}_{n}$ for all $n \in \mathcal{N}$, where $\zeta_{n}$ is an arbitrary nonnegative weight associated with $n$th secondary RX. Weights $\zeta_{n}$ can be used to balance between user fairness and total throughput.

${ }^{\mathrm{e}}$ Note that in worst-case robust optimization, the beamforming vectors are designed to keep interference to the primary RXs below the threshold for all possible channel errors (irrespective of their probability of occurrence) inside the uncertainty region. In the simulations, each complex entry of the channel error vector is generated using a truncated Gaussian distribution $C N\left(0, \zeta^{2}\right)$ truncated at $-3 \zeta$ and $3 \zeta$ (roughly speaking, this corresponds to $1 \%$ error outage), where $\zeta=\xi / 3 \sqrt{2 n_{t}}$ denotes the standard deviation. The larger value of $\xi$ implies that the actual channel vector can be very far from the estimated value of the channel. Since in the considered simulation model, the probability of occurrence of the channel vectors which are far from the estimated value is small the probability of achieving interference close to the threshold is small.

${ }^{\mathrm{f}}$ Note that in the non-robust design, there is no null present at $\theta=90^{\circ}$. This is due to the fact that the beamforming design assumes perfect CSI at the secondary TX. Since the secondary TX knows the exact channel to the primary RX, even with this gain, the secondary TX can guarantee that the interference to the primary RX for that specific estimated channel is below the interference threshold.

\section{Appendices}

\section{Appendix 1}

\section{Proof of proposition 1}

Let us rewrite Problem (23) as follows:

$$
\begin{aligned}
& \text { minimize } \sum_{n=1}^{N} \operatorname{Trace}\left(\mathbf{W}_{n}\right) \\
& \text { subject to } \\
& \boldsymbol{\Delta}_{n n}\left(\mathbf{W}_{n}, \gamma, \mu_{n n},\left\{s_{m n}\right\}_{m \neq n}\right) \succeq 0, \quad n \in \mathcal{N} \\
& \boldsymbol{\Phi}_{m n}\left(\mathbf{W}_{m}, \mu_{m n}, s_{m n}\right) \geq 0, m \in \mathcal{N} \backslash\{n\}, n \in \mathcal{N} \\
& \boldsymbol{\Theta}_{n k}\left(\mathbf{W}_{n}, v_{n k}, I_{n k}\right) \succeq 0, \quad n \in \mathcal{N}, k \in \mathcal{K} \\
& \mathbf{W}_{n} \geq 0, \quad n \in \mathcal{N} \\
& \text { Trace }\left(\mathbf{W}_{n}\right) \leq P_{\max }, \quad n \in \mathcal{N} \\
& \sum_{n=1}^{N} I_{n k} \leq I_{\mathrm{th}}, \quad k \in \mathcal{K} \\
& \mu_{m n} \geq 0, \quad n, m \in \mathcal{N} \\
& v_{n k} \geq 0, \quad n \in \mathcal{N}, k \in \mathcal{K}
\end{aligned}
$$

where the optimization variables are $\mathbf{W}_{n}, \mu_{m n}, v_{n k}, s_{m n}, I_{n k}$ for all $n, m \in \mathcal{N}, k \in \mathcal{K}$ for a fixed $\gamma$ value. The corresponding dual variables for the constraints are shown in the rightmost column of Problem (44).

Let $\mathcal{L}(\cdot)$ define the Lagrangian of Problem (44) and

$$
\boldsymbol{\Psi}_{n n}=\left[\begin{array}{cc}
\mathbf{A}_{n n} & \mathbf{b}_{n n} \\
\mathbf{b}_{n n}^{H} & c_{n n}
\end{array}\right] \succeq 0 \quad n \in \mathcal{N}
$$

where $\mathbf{A}_{n n} \in \mathbb{H}^{n_{t}}, \mathbf{b}_{n n} \in \mathbb{C}^{n_{t}}$ and $c_{n n}$ is a non-negative real value according to the properties of positive semidefinite matrices. Using the Karush-Kuhn-Tucker (KKT) conditions, gradient $(\nabla)$, complimentary slackness (C.S.), primal feasibility (P.F.) and dual feasibility (D.F.), we can write the following:

$$
\begin{aligned}
& \nabla \mathcal{L}_{\mathbf{W}_{n}^{\star}}=0 \Rightarrow \mathbf{Z}_{n}^{\star}=\left(1+\beta_{n}^{\star}\right) \mathbf{I}_{n_{t}}+\sum_{\substack{m=1 \\
m \neq n}}^{N}\left[\mathbf{I}_{n_{t}} \hat{\mathbf{g}}_{n m}\right] \boldsymbol{\Psi}_{n m}^{\star}\left[\begin{array}{c}
\mathbf{I}_{n_{t}} \\
\hat{\mathbf{g}}_{n m}^{H}
\end{array}\right] \\
& +\sum_{k=1}^{K}\left[\begin{array}{ll}
\mathbf{I}_{n_{t}} & \hat{\mathbf{h}}_{n k}
\end{array}\right] \boldsymbol{\Lambda}_{n k}^{\star}\left[\begin{array}{c}
\mathbf{I}_{n_{t}} \\
\hat{\mathbf{h}}_{n k}^{H}
\end{array}\right] \\
& -\frac{1}{\gamma}\left[\mathbf{I}_{n_{t}} \hat{\mathbf{g}}_{n n}\right] \boldsymbol{\Psi}_{n n}^{\star}\left[\begin{array}{c}
\mathbf{I}_{n_{t}} \\
\hat{\mathbf{g}}_{n n}^{H}
\end{array}\right], \quad n \in \mathcal{N}
\end{aligned}
$$

$\nabla \mathcal{L}_{\mu_{n n}^{\star}}=0 \Rightarrow \operatorname{Trace}\left(\mathbf{Q}_{n n} \mathbf{A}_{n n}^{\star}\right)-c_{n n}^{\star}+\eta_{n n}^{\star}=0, n \in \mathcal{N}$

$$
\begin{array}{lr}
\text { C.S. } \mathbf{W}_{n}^{\star} \succeq 0 & \mathbf{Z}_{n}^{\star} \mathbf{W}_{n}^{\star}=\mathbf{0}, \quad n \in \mathcal{N} \\
\text { C.S. } \Delta_{n n}^{\star} \succeq 0 \Rightarrow & \boldsymbol{\Delta}_{n n}^{\star} \mathbf{\Psi}_{n n}^{\star}=\mathbf{0}, \quad n \in \mathcal{N} \\
\text { P.F. } \mu_{m n}^{\star} \Rightarrow & \mu_{m n}^{\star} \geq 0, \quad n, m \in \mathcal{N} \\
\text { P.F. } \Phi_{m n}^{\star} \Rightarrow & \Phi_{m n}^{\star} \succeq 0, \quad n, m \in \mathcal{N} \\
\text { D.F. } \mathbf{Z}_{n}^{\star} \Rightarrow & \mathbf{Z}_{n}^{\star} \succeq 0, \quad n \in \mathcal{N} \\
\text { D.F. } \Psi_{m n}^{\star} \Rightarrow & \boldsymbol{\Psi}_{m n}^{\star} \succeq 0, \quad n, m \in \mathcal{N} \\
\text { D.F. } \Lambda_{n k}^{\star} \Rightarrow & \mathbf{\Lambda}_{n k}^{\star} \succeq 0, \quad n \in \mathcal{N}, k \in \mathcal{K} \\
\text { D.F. } \eta_{m n}^{\star} \Rightarrow & \eta_{m n}^{\star} \geq 0, \quad n, m \in \mathcal{N} \\
\text { D.F. } \beta_{n}^{\star} \Rightarrow & \beta_{n}^{\star} \geq 0, \quad n \in \mathcal{N} .
\end{array}
$$

Further, we can observe for Problem (44) that the target minimum SINR

$$
\gamma>0 \Rightarrow \mathbf{W}_{n}^{\star} \neq 0, \quad n \in \mathcal{N} .
$$


Now, we write the first-three term of (46) as

$$
\begin{aligned}
\overline{\mathbf{Z}}_{n}^{\star}= & \left(1+\beta_{n}^{\star}\right) \mathbf{I}_{n_{t}}+\sum_{\substack{m=1 \\
m \neq n}}^{N}\left[\mathbf{I}_{n_{t}} \hat{\mathbf{g}}_{n m}\right] \boldsymbol{\Psi}_{n m}^{\star}\left[\begin{array}{c}
\mathbf{I}_{n_{t}} \\
\hat{\mathbf{g}}_{n m}^{H}
\end{array}\right] \\
& +\sum_{k=1}^{K}\left[\mathbf{I}_{n_{t}} \hat{\mathbf{h}}_{n k}\right] \boldsymbol{\Lambda}_{n k}^{\star}\left[\begin{array}{c}
\mathbf{I}_{n_{t}} \\
\hat{\mathbf{h}}_{n k}^{H}
\end{array}\right] .
\end{aligned}
$$

From (53), (54) and (56), we can say that

$$
\overline{\mathbf{Z}}_{n}^{\star} \succ 0 \Rightarrow \operatorname{Rank}\left(\overline{\mathbf{Z}}_{n}^{\star}\right)=n_{t},
$$

since $\overline{\mathbf{Z}}_{n}^{\star}$ should be full ranked. Moreover, if $\boldsymbol{\Psi}_{n n}^{\star}=\mathbf{0}$, (46) and (59) suggest that $\mathbf{Z}_{n}^{\star} \succ \mathbf{0}$. From (48), this leads to $\mathbf{W}_{n}^{\star}=\mathbf{0}$ which contradicts with (57). Therefore, the condition

$$
\boldsymbol{\Psi}_{n n}^{\star} \neq \mathbf{0}, \quad n \in \mathcal{N}
$$

should be satisfied.

The rest of the proof follows from the proof of proposition 1 in [18] and we provide the details for the completeness of the paper. First, we prove that $\mathbf{W}_{n}^{\star}$ has rank one provided that $\Psi_{n n}^{\star}$ has rank one. Then we prove that indeed $\Psi_{n n}^{\star}$ has rank one.

Assume that $\boldsymbol{\Psi}_{n n}^{\star}$ has rank one and therefore it can be written as $\boldsymbol{\Psi}_{n n}^{\star}=\mathbf{v v}^{H}$, where $\mathbf{v} \in \mathbb{C}^{n_{t}+1}$. Then the last term in (46) can be written as $\tilde{\mathbf{Z}}_{n}^{\star}=$

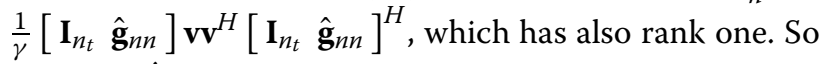
we can write

$$
\begin{aligned}
\operatorname{Rank}\left(\overline{\mathbf{Z}}_{n}^{\star}\right) & =\operatorname{Rank}\left(\mathbf{Z}_{n}^{\star}+\tilde{\mathbf{Z}}_{n}^{\star}\right) \\
& \leq \operatorname{Rank}\left(\mathbf{Z}_{n}^{\star}\right)+\operatorname{Rank}\left(\tilde{\mathbf{Z}}_{n}^{\star}\right) \\
\Rightarrow n_{t}-1 & \leq \operatorname{Rank}\left(\mathbf{Z}_{n}^{\star}\right) .
\end{aligned}
$$

Using Sylvester's rank inequality (see p. 211 in [39]) on (48) and with the help of (61), we can write following for rank of $\mathbf{W}_{n}^{\star}$ :

$$
\operatorname{Rank}\left(\mathbf{W}_{n}^{\star}\right) \leq n_{t}+\operatorname{Rank}\left(\mathbf{Z}_{n}^{\star} \mathbf{W}_{n}^{\star}\right)-\operatorname{Rank}\left(\mathbf{Z}_{n}^{\star}\right) \leq 1 .
$$

It follows from (57) and (62) that

$$
\operatorname{Rank}\left(\mathbf{W}_{n}^{\star}\right)=1 \text {. }
$$

What remains is to prove that $\Psi_{n n}^{\star}$ indeed has rank one. First, we will show that $c_{n n}^{\star}>0$ and $\mu_{n n}^{\star}>0$. From (47) and (55), we can write

$$
\text { Trace }\left(\mathbf{Q}_{n n} \mathbf{A}_{n n}^{\star}\right) \leq c_{n n}^{\star}, \quad n \in \mathcal{N} .
$$

Since $\mathbf{Q}_{n n} \succ 0$, by (45), (60) and (64), it is obvious that $c_{n n}^{\star}>0$. We can see from (18) that when $\mu_{n n}=0, \boldsymbol{\Delta}_{n n}$ is not positive semidefinte because

$$
\begin{aligned}
& {\left[\begin{array}{ll}
-\hat{\mathbf{g}}_{n n}^{H} & 1
\end{array}\right] \boldsymbol{\Delta}_{n n}\left(\mathbf{W}_{n}^{\star}, \gamma, \mu_{n n}^{\star},\left\{s_{m n}^{\star}\right\}_{m \neq n}\right)\left[\begin{array}{c}
-\hat{\mathbf{g}}_{n n} \\
1
\end{array}\right]} \\
& \quad=-\gamma\left(\sum_{\substack{m=1 \\
m \neq n}}^{N} s_{m n}^{\star}+\sigma_{n}^{2}\right)<0 .
\end{aligned}
$$

This implies that $\mu_{n n} \neq 0$ and hence from (50), we can say that $\mu_{n n}>0$.

By substituting (18) and (45) into (49), the following two equalities can be easily obtained:

$$
\left(\mathbf{W}_{n}^{\star}+\mu_{n n}^{\star} \mathbf{Q}_{n n}\right) \mathbf{A}_{n n}^{\star}+\mathbf{W}_{n}^{\star} \hat{\mathbf{g}}_{n n} \mathbf{b}_{n n}^{\star H}=\mathbf{0},
$$

$$
\left(\mathbf{W}_{n}^{\star}+\mu_{n n}^{\star} \mathbf{Q}_{n n}\right) \mathbf{b}_{n n}^{\star}+\mathbf{W}_{n}^{\star} \hat{\mathbf{g}}_{n n} c_{n n}^{\star}=\mathbf{0} .
$$

Since $c_{n n}>0$, post-multiplying (67) by $-\mathbf{b}_{n n}^{H} / c_{n n}$ and adding to (66) result in

$$
\left(\mathbf{W}_{n}^{\star}+\mu_{n n}^{\star} \mathbf{Q}_{n n}\right)\left(\mathbf{A}_{n n}^{\star}-\mathbf{b}_{n n}^{\star} \mathbf{b}_{n n}^{\star H} / c_{n n}^{\star}\right)=\mathbf{0} .
$$

The term $\left(\mathbf{W}_{n}^{\star}+\mu_{n n}^{\star} \mathbf{Q}_{n n}\right) \succ \mathbf{0}$, since $\mu_{n n}^{\star}>0$ and $\mathbf{Q}_{n n} \succ$ 0, then (68) implies that $\mathbf{A}_{n n}^{\star}=\mathbf{b}_{n n}^{\star} \mathbf{b}_{n n}^{\star} H / c_{n n}^{\star}$. Hence, (45) transforms to

$\boldsymbol{\Psi}_{n n}^{\star}=\left[\begin{array}{cc}\mathbf{b}_{n n}^{\star} \mathbf{b}_{n n}^{\star H} / c_{n n}^{\star} & \mathbf{b}_{n n}^{\star} \\ \mathbf{b}_{n n}^{\star H} & c_{n n}^{\star}\end{array}\right]=\left[\begin{array}{c}\mathbf{b}_{n n}^{\star} / \sqrt{c_{n n}^{\star}} \\ \sqrt{c_{n n}^{\star}}\end{array}\right]\left[\mathbf{b}_{n n}^{\star H} / \sqrt{c_{n n}^{\star}} \sqrt{c_{n n}^{\star}}\right]$,

which is indeed a rank-one matrix. This concludes the proof.

\section{Appendix 2}

\section{Proof of feasibility of eigenvalue approximation}

Consider a positive definite matrix $\mathbf{Y} \in \mathbb{H}^{l}$ with $r=$ $\operatorname{Rank}(\mathbf{Y})$. Then according to the eigenvalue decomposition, we can write

$$
\mathbf{Y}=\sum_{i=1}^{r} \lambda_{i} \mathbf{y}_{i} \mathbf{y}_{i}^{H}
$$

where $\lambda_{1} \geq \lambda_{2} \geq \ldots \geq \lambda_{r}>0$ are the eigenvalues and $\mathbf{y}_{1}, \ldots, \mathbf{y}_{r} \in \mathbb{C}^{l}$ are the respective eigenvectors of $\mathbf{Y}$. Since $\mathbf{Y}$ is positive semidefinite, for any random vector $\mathbf{z} \in \mathbb{C}^{l}$,

$$
\mathbf{z}^{H} \mathbf{Y} \mathbf{z} \geq \lambda_{1} \mathbf{z}^{H} \mathbf{y}_{1} \mathbf{y}_{1}^{H} \mathbf{z} \geq 0
$$

Here, the first inequality is achieved from the fact that all the eigenvalues are non-negative and the definition of positive semidefinite matrix gives the second inequality.

Further, consider any vector $\mathbf{z} \in \mathbb{C}^{l}$ where the elements are denoted by $z_{1}, \ldots, z_{n} \in \mathbb{C}$. Then, from the definition

$$
\operatorname{Trace}\left(\mathbf{z z}^{H}\right)=\sum_{i=1}^{l} z_{i} z_{i}^{H} \geq 0
$$

Let the solution of relaxed SDP (29) be given by $\gamma^{\star}, x^{\star}$ and $\mathbf{V}_{n}^{\star}, \check{\mu}_{m n}^{\star}, \check{v}_{n k}^{\star}, u_{m n}^{\star}, y_{n k}^{\star}$ for all $n, m \in \mathcal{N}, k \in \mathcal{K}$. Now, from the perturbed Problem (28), we can say that for the 
matrices $\mathbf{V}_{n}^{\star}$ for all $n \in \mathcal{N}$ there exists a $\gamma^{\star} \geq 0$ such that the following conditions are satisfied:

$$
\left(\hat{\mathbf{g}}_{n n}^{H}+\mathbf{e}_{n n}^{H}\right) \mathbf{V}_{n}^{\star}\left(\hat{\mathbf{g}}_{n n}+\mathbf{e}_{n n}\right) \geq \gamma^{\star}, \quad \forall \mathbf{e}_{n n}^{H} \mathbf{Q}_{n n} \mathbf{e}_{n n} \leq 1, \quad n \in \mathcal{N}
$$

$$
\begin{aligned}
& \sum_{\substack{m=1 \\
m \neq n}}^{N} u_{m n}^{\star}+\sigma_{n}^{2} x^{\star} \leq 1, \quad n \in \mathcal{N} \\
&\left(\hat{\mathbf{g}}_{m n}^{H}+\mathbf{e}_{m n}^{H}\right) \mathbf{V}_{m}^{\star}\left(\hat{\mathbf{g}}_{m n}+\mathbf{e}_{m n}\right) \leq u_{m n}^{\star}, \quad \forall \mathbf{e}_{m n}^{H} \mathbf{Q}_{m n} \mathbf{e}_{m n} \leq 1, \\
& n, m \in \mathcal{N}
\end{aligned}
$$

$$
\begin{aligned}
& \sum_{n=1}^{N} y_{n k}^{\star} \leq I_{\mathrm{th}} x^{\star}, \quad k \in \mathcal{K} \\
& \left(\hat{\mathbf{h}}_{n k}^{H}+\tilde{\mathbf{e}}_{n k}^{H}\right) \mathbf{V}_{n}^{\star}\left(\hat{\mathbf{h}}_{n k}+\tilde{\mathbf{e}}_{n k}\right) \leq y_{n k}^{\star}, \quad \forall \tilde{\mathbf{e}}_{n k}^{H} \tilde{\mathbf{Q}}_{n k} \tilde{\mathbf{e}}_{n k} \leq 1, \\
& n \in \mathcal{N}, \quad k \in \mathcal{K} \\
& \operatorname{Tr}\left(\mathbf{V}_{n}^{\star}\right) \leq P_{\max } x^{\star}, \quad n \in \mathcal{N} .
\end{aligned}
$$

Therefore, in order to show that the eigenvalue approximation is feasible, we should be able to find a $\gamma \geq 0$ for the approximated beamforming vectors such that all above inequalities are satisfied.

Let the rank of $\mathbf{V}_{n}^{\star}$ be $r$, eigenvalues and the corresponding eigenvectors be $\lambda_{1, n} \geq \lambda_{2, n} \geq \ldots, \geq \lambda_{r, n} \geq 0$ and $\mathbf{y}_{1, n}, \mathbf{y}_{2, n}, \ldots, \mathbf{y}_{r, n}$ respectively for all $n \in \mathcal{N}$. Then, using the eigenvalue decomposition, the trace of the beamforming matrix can be written as

$$
\operatorname{Tr}\left(\mathbf{V}_{n}^{\star}\right)=\sum_{j=1}^{r} \operatorname{Tr}\left(\lambda_{j, n} \mathbf{y}_{j, n} \mathbf{y}_{j, n}^{H}\right) \geq \operatorname{Tr}\left(\lambda_{1, n} \mathbf{y}_{1, n} \mathbf{y}_{1, n}^{H}\right),
$$

where the inequality is obtained using (72) and the fact that all eigenvalues are non-negative. Therefore, it can be easily shown from (74) that the condition (73f) is satisfied for the eigenvalue approximation.

Then from (71), for any error vector $\tilde{\mathbf{e}}_{n k}$ we can say that,

$$
\begin{gathered}
\left(\hat{\mathbf{h}}_{n k}^{H}+\tilde{\mathbf{e}}_{n k}^{H}\right) \lambda_{1, n} \mathbf{y}_{1, n} \mathbf{Y}_{1, n}^{H}\left(\hat{\mathbf{h}}_{n k}+\tilde{\mathbf{e}}_{n k}\right) \\
\leq\left(\hat{\mathbf{h}}_{n k}^{H}+\tilde{\mathbf{e}}_{n k}^{H}\right) \mathbf{V}_{n}^{\star}\left(\hat{\mathbf{h}}_{n k}+\tilde{\mathbf{e}}_{n k}\right) .
\end{gathered}
$$

This implies that the condition (73e) is satisfied with the approximation. Further, the same method can be applied to prove that the condition $(73 \mathrm{c})$ is also satisfied.
According to (71), in order to satisfy the condition (73a), the beamforming matrix $\mathbf{V}_{n}^{\star}$ should be rank one for all $n \in \mathcal{N}$, i.e., when the first inequality of (71) becomes an equality. But from the second inequality of (71), we can say that for any error vector $\mathbf{e}_{n n}$ there exists some $\gamma^{\text {new }}$, such that

$$
\begin{aligned}
\gamma^{\star} & \geq\left(\hat{\mathbf{g}}_{n n}^{H}+\mathbf{e}_{n n}^{H}\right) \lambda_{1, n} \mathbf{y}_{1, n} \mathbf{y}_{1, n}^{H}\left(\hat{\mathbf{g}}_{n n}+\mathbf{e}_{n n}\right) \geq \gamma^{\text {new }} \geq 0 \\
n & \in \mathcal{N} .
\end{aligned}
$$

This concludes the proof and shows that we can find a $\gamma^{\text {new }} \geq 0$ for the eigenvalue approximation such that the problem is feasible, but which will be a lower bound for the solution.

\section{Competing interests}

The authors declare that they have no competing interests.

\section{Author details}

${ }^{1}$ Centre for Wireless Communications, University of Oulu, P.O. Box 4500, Oulu 90014, Finland. ${ }^{2}$ University of Maryland, College Park, MD 20742, USA.

Received: 06 August 2013 Accepted: 03 March 2014

Published: 12 March 2014

\section{References}

1. FCC, Spectrum policy task force report. Tech. Rep. TR 02-155 (2002). http://www.fcc.gov/sptf/reports.html. Accessed 15 March 2013

2. J Mitola III, Cognitive radio: an integrated agent architecture for software defined radio. PhD thesis, Royal Institute of Technology, Sweden, 2000

3. S Haykin, Cognitive radio: brain-empowered wireless communications. IEEE J. Selected Areas Commun. 23(2), 201-220 (2005)

4. A Goldsmith, SA Jafar, I Maric, S Srinivasa, Breaking spectrum gridlock with cognitive radios: an information theoretic perspective. Proc. IEEE 97(5), 894-914 (2009)

5. SJ Kim, GB Giannakis, Optimal resource allocation for MIMO ad hoc cognitive radio networks, in Proceedings of the Annual Allerton Conference on Communications, Control, and Computing, 23-26 September 2008 (Monticello, IL, 2008), pp. 39-45

6. G Scutari, DP Palomar, S Barbarossa, Competitive optimization of cognitive radio MIMO systems via game theory, in Proceedings of the International Conference on Game Theory for Networks (Istanbul, Turkey, 2009), pp. 452-461

7. H Islam, YC Liang, A Hoang, Joint power control and beamforming for cognitive radio networks. IEEE Trans. Wireless Commun. 7(7), 2415-2419 (2008)

8. L Zhang, R Zhang, YC Liang, Y Xin, HV Poor, On Gaussian MIMO BC-MAC duality with multiple transmit covariance constraints. IEEE Trans. Inf. Theory 58(4), 2064-2078 (2012)

9. L Zhang, Y Xin, YC Liang, Weighted sum rate optimization for cognitive radio MIMO broadcast channels. IEEE Trans. Wireless Commun. 8(6), 2950-2959 (2009)

10. K Yang, Y Wu, J Huang, X Wang, S Verdu, Distributed robust optimization for communication networks, in Proceedings of the IEEE International Conference on Computer Communications, 15-17 April 2008 (Phoenix, AZ, 2008), pp. 1157-1165

11. M Shenouda, TN Davidson, On the design of linear transceivers for multiuser systems with channel uncertainty. IEEE J. Selected Areas Commun. 26(6), 1015-1024 (2008)

12. E Song, Q Shi, M Sanjabi, R Sun, ZQ Luo, Robust SINR-constrained MISO downlink beamforming: when is semidefinite programming relaxation tight?, in Proceedings of the IEEE International Conference on Acoustics, 
Speech, and Signal Processing, 22-27 May 2011 (Prague, Czech Republic, 2011), pp. 3096-3099

13. SJ Kim, A Magnani, A Mutapcic, SP Boyd, ZQ Luo, Robust beamforming via worst-case SINR maximization. IEEE Trans. Signal Process. 56(4), 1539-1547 (2008)

14. RG Lorenz, SP Boyd, Robust minimum variance beamforming. IEEE Trans. Signal Process. 53(5), 1684-1696 (2005)

15. E Björnson, $G$ Zheng, M Bengtsson, B Ottersten, Robust monotonic optimization framework for Multicell MISO systems. IEEE Trans. Signal Process. 60(5), 2508-2523 (2012)

16. E Björnson, E Jorswieck, Optimal resource allocation in coordinated multi-cell systems. Foundations Trends Commun. Inf. Theory $\mathbf{9}(2-3)$, 111-381 (2013)

17. A Mutapcic, SJ Kim, S Boyd, A Tractable Method for Robust Downlink Beamforming in Wireless Communications, in Proceedings of the Annual Asilomar Conference on Signals, Systems and Computers, 4-7 November 2007 (Pacific Grove, CA, 2007), pp. 1224-1228

18. C Shen, TH Chang, KY Wang, Z Qiu, CY Chi, Distributed robust multicell coordinated beamforming with imperfect CSI: an ADMM Approach. IEEE Trans. Signal Process. 60(6), 2988-3003 (2012)

19. SA Vorobyov, AB Gershman, ZQ Luo, Robust adaptive beamforming using worst-case performance optimization: a solution to the signal mismatch problem. IEEE Trans. Signal Process. 51(2), 313-324 (2003)

20. F Wang, W Wang, Robust beamforming and power control for multiuser cognitive radio network, in Proceedings of the IEEE Global Telecommunication Conference, 6-10 December 2010 (Miami, FL, 2010), pp. 1-5

21. G Zheng, KK Wong, B Ottersten, Robust cognitive beamforming with bounded channel uncertainties. IEEE Trans. Signal Process. 57(12), 4871-4881 (2009)

22. MF Hanif, PJ Smith, M Alouini, SINR balancing in the downlink of cognitive radio networks with imperfect channel knowledge, in Proceedings of the IEEE International Conference on Cognitive Radio Oriented Wireless Networks and Communications, 9-11 June 2010 (Cannes, France, 2010), pp. 1-5

23. I Wajid, M Pesavento, YC Eldar, A Gershman, Robust downlink beamforming for cognitive radio networks, in Proceedings of the IEEE Global Telecommunication Conference, 6-10 December 2010 (Miami, FL, 2010), pp. 1-5

24. EA Gharavol, Y Liang, K Mouthaan, Robust linear transceiver design in MIMO ad hoc cognitive radio networks with imperfect channel state information. IEEE Trans. Wireless Commun. 10(5), 1448-1457 (2011)

25. Y Huang, Q Li, WK Ma, S Zhang, Robust multicast beamforming for spectrum sharing-based cognitive radios. IEEE Trans. Signal Process. 60, 527-533 (2012)

26. Y Zhang, ED Anese, GB Giannakis, Distributed optimal beamformers for cognitive radios robust to channel uncertainties. IEEE Trans. Signal Process. 60(12), 6495-6508 (2012)

27. L Zhang, YC Liang, Y Xin, HV Poor, Robust cognitive beamforming with partial channel state information. IEEE Trans. Wireless Commun. 8(8), 4143-4153 (2009)

28. G Zheng, S Ma, KK Wong, TS Ng, Robust beamforming in cognitive radio. IEEE Trans. Wireless Commun. 9(2), 570-576 (2010)

29. ZQ Luo, WK Ma, AMC So, Y Ye, S Zhang, Semidefinite relaxation of quadratic optimization problems. IEEE Signal Process. Mag. 27(3), 20-34 (2010)

30. YJ Zhang, AMC So, Optimal spectrum sharing in MIMO cognitive radio networks via semidefinite programming. IEEE J. Selected Areas Commun. 29(2), 362-373 (2011)

31. S Boyd, L Vandenberghe, Convex Optimization. (Cambridge University Press, Cambridge, 2004)

32. UT Jönsson, A Lecture on the S-procedure (2001). http://www.math.kth. se/ ulfj/5B5746/Lecture.ps. Accessed 12 January 2013

33. CP Robert, Simulation of truncated normal variables. Springer J. Stat. Comput. 5(2), 121-125 (1995)

34. DJ Olive, Applied robust statistics. Preprint M-02-006 (2008). http:// lagrange.math.siu.edu/Olive/ol-bookp.htm. Accessed 1 March 2013

35. M Botros, TN Davidson, Convex conic formulations of robust downlink precoder designs with quality of service constraints. IEEE J. Selected Top. Signal Process. 1(4), 714-724 (2007)

36. I Polik, SeDuMi (2010). http://sedumi.ie.lehigh.edu/. Accessed 25 January 2013
37. KC Toh, MJ Todd, RH Tütüncü, SDPT3 - A Matlab software package for semidefinite programming, Version 1.3. Optimization Methods Softw. 11(1-4), 545-581 (1999)

38. CVX Research I, CVX: Matlab Software for Disciplined Convex Programming (2012). http://cvxr.com/cvx. Accessed 25 January 2013

39. CD Meyer, Matrix Analysis and Applied Linear Algebra. (Society for Industrial and Applied Mathematics, Philadelphia, PA, 2000)

doi:10.1186/1687-1499-2014-37

Cite this article as: Wijewardhana et al:: A robust beamformer design for underlay cognitive radio networks using worst case optimization. EURASIP Journal on Wireless Communications and Networking 2014 2014:37.

\section{Submit your manuscript to a SpringerOpen ${ }^{\circ}$ journal and benefit from:}

- Convenient online submission

Rigorous peer review

- Immediate publication on acceptance

- Open access: articles freely available online

- High visibility within the field

- Retaining the copyright to your article

Submit your next manuscript at $\boldsymbol{~ s p r i n g e r o p e n . c o m ~}$ 